\title{
Incidence du cancer chez les enfants au Canada : variations démographiques et géographiques des tendances (1992-2010)
}

\author{
Lin Xie, M. Sc.; Jay Onysko, M. A.; Howard Morrison, Ph. D.
}

Cet article de recherche quantitative a fait l'objet d'une évaluation par les pairs.

\section{Résumé}

Introduction : La surveillance des tendances de l'incidence du cancer chez les enfants peut éclairer la recherche, les politiques et les programmes en matière d'étiologie. Cette étude donne lieu au premier rapport sur les variations démographiques et géographiques des tendances de l'incidence de groupes diagnostiques détaillés chez les enfants de la population générale au Canada.

Méthodologie : Les données du Registre canadien du cancer ont servi à calculer les taux d'incidence normalisés selon l'âge (TINA) annuels de 1992 à 2010 chez les enfants de moins de 15 ans selon le sexe, l'âge et la région pour les 12 principaux groupes et certains sous-groupes diagnostiques de la $3^{\mathrm{e}}$ édition de la Classification internationale du cancer chez les enfants (CICE). Les tendances temporelles ont été examinées à partir des variations annuelles en pourcentage (VAP) au moyen d'une régression Joinpoint.

Résultats : Les TINA annuels du cancer chez les enfants ont augmenté de 0,5\% (intervalle de confiance [IC] à $95 \%=0,2$ à 0,9$)$ chez les garçons chaque année entre 1992 et 2010, tandis que l'incidence chez les filles a augmenté de $3,2 \%$ (IC $=0,4$ à 6,2) chaque année depuis 2004 après une stabilisation initiale. L'augmentation globale la plus importante a été observée chez les enfants de 1 à 4 ans (VAP $=0,9 \%$, IC $=0,4$ à 1,3). Par région, c'est en Ontario entre 2006 et 2010 que les taux globaux ont le plus augmenté $(\mathrm{VAP}=5,9 \%, \mathrm{IC}=1,9$ à 10,1$)$ et ils ont augmenté de façon non significative dans les autres régions entre 1992 et 2010. On a mesuré en 2006-2010 les TINA annuels moyens de tous les cancers confondus les plus faibles dans les Prairies (149,4 pour 1 million) et les plus élevés en Ontario (170,1 pour 1 million). Les TINA des leucémies, du mélanome, des carcinomes, du cancer de la thyroïde, des épendymomes et de l'hépatoblastome ont augmenté dans tous les groupes d'âge, et les TINA du neuroblastome ont augmenté chez les enfants de 1 à 4 ans. L'incidence de l'astrocytome a diminué chez les enfants de 10 à 14 ans (VAP $=-2,1 \%, \mathrm{IC}=-3,7$ à $-0,5)$ ainsi que chez les garçons (VAP $=-2,4 \%$, $\mathrm{IC}=-4,6$ à $-0,2)$ et les filles (VAP $=-3,7 \%$, IC $=-5,8$ à $-1,6$ ) en Ontario au cours de la période étudiée.

Conclusion : Les tendances à la hausse de l'incidence de tous les cancers confondus et de certaines tumeurs malignes correspondent aux tendances signalées dans d'autres pays développés. Elles sont explicables par des variations démographiques, par des variations de l'exposition aux facteurs étiologiques ou par des changements apportés aux méthodes de codification, de diagnostic et de déclaration du cancer. Une baisse significative de la tendance de l'astrocytome chez les 10 à 14 ans a été observée pour la première fois.

Mots-clés : cancer chez l'enfant, CICE, taux d'incidence normalisé selon l'âge, variation annuelle en pourcentage

\section{Introduction}

Bien que le cancer chez les enfants soit rare et constitue moins de $1 \%$ de tous les nouveaux cas de cancer au Canada, il est la cause la plus courante de décès (après les accidents) chez les enfants de plus d'un an au Canada ${ }^{1,2}$. Même si les progrès dans les traitements ont permis d'augmenter le taux global de survie à 5 ans, qui est passé

\section{Points saillants}

- L’incidence du cancer chez les enfants a augmenté de 0,5\% par année entre 1992 et 2010 chez les garçons et de 3,2 \% entre 2004 et 2010 chez les filles.

- On a observé au cours des dix dernières années une augmentation globale et une augmentation chez les enfants de 1 à 4 ans.

- L'incidence globale a augmenté de manière générale dans chaque région entre 1992 et 2010. Les taux les plus faibles correspondaient aux Prairies et les plus élevés à l'Ontario pour la période de 2006 à 2010.

- Des hausses significatives des leucémies, du mélanome, des carcinomes, du cancer de la thyroïde, des épendymomes et de l'hépatoblastome ont été observées dans tous les groupes d'âge combinés, et une hausse significative du neuroblastome a été constatée chez les enfants de 1 à 4 ans.

- L'incidence de l'astrocytome a diminué chez les enfants de 10 à 14 ans.

- Ces résultats sont susceptibles de contribuer à éclairer la recherche ainsi que les politiques et les programmes de santé publique en matière d'étiologie.

de $71 \%$ à $83 \%$ en 30 ans, le cancer chez les enfants a des répercussions à vie sur la santé, l'état psychosocial et la situation financière de ces enfants et de leurs familles $^{1,3}$. Les patients qui survivent 5 ans risquent de voir leur cancer primitif récidiver ou progresser et risquent davantage de développer plus tard des tumeurs malignes, des maladies chroniques et des incapacités fonctionnelles en raison des traitements. 
Un rapport de Statistique Canada a fait état d'une augmentation statistiquement significative de 0,4\% par année de l'incidence globale des cancers chez les enfants entre 1992 et 2010 à l'échelle nationale ${ }^{4}$. Au cours des dernières années, la possibilité que les taux d'incidence de certaines tumeurs malignes de l'enfant augmentent est devenue un sujet de préoccupation pour la population et pour la communauté scientifique $^{5-8}$. Les raisons de ces variations ne sont pas encore comprises. La surveillance des tendances de l'incidence du cancer pourrait permettre de poser de nouvelles hypothèses pour les futures études étiologiques et révéler des besoins en services de santé pour des populations spécifiques. Or les tendances temporelles récentes de l'incidence n'ont jusqu'à présent jamais été examinées en détail par groupes diagnostiques chez l'enfant ou en fonction du contexte régional. Cette étude fournit des données populationnelles détaillées et récentes sur les variations démographiques et géographiques observées dans les tendances de l'incidence du cancer chez les enfants au Canada.

\section{Méthodologie}

\section{Sources de données}

Les données sur l'incidence du cancer ont été tirées du Registre canadien du cancer $(\mathrm{RCC})^{9}$, sauf pour le Québec où, pour 2008 à 2010, les données ont été obtenues directement de la province sous forme synthétique. Les données sur l'incidence sont recueillies par les registres provinciaux et territoriaux du cancer puis transmises chaque année au RCC de Statistique Canada. Le RCC est une base de données populationnelles dynamique et axée sur les personnes qui recense les nouveaux cas diagnostiqués depuis 1992.

Les diagnostics de cancer ont été codés selon la topographie, la morphologie et le comportement à l'aide de la $3^{\text {e }}$ édition de la Classification internationale des maladies pour l'oncologie (CIM-O-3) ${ }^{10}$ et ont été convertis pour correspondre à la $3^{\mathrm{e}}$ édition de la Classification internationale du cancer chez les enfants (CICE-3) ${ }^{11,12}$. Tous les cancers primitifs diagnostiqués entre 1992 et 2010 chez les enfants de la naissance à 14 ans ont été inclus. La CICE-3 classe les tumeurs intracrâniennes et intramédullaires non malignes dans les catégories III et X. Conformément à cette classification, les tumeurs non malignes du système nerveux central (SNC) ont été incluses dans une analyse distincte.

Les estimations de la population du Canada et des provinces et territoires utilisées dans le calcul des taux d'incidence reposent sur les recensements quinquennaux menés de 1991 à 2011. Nous avons utilisé les estimations intercensitaires établies par Statistique Canada pour les années entre chaque recensement ${ }^{13}$.

\section{Analyse statistique}

Les données sur l'incidence du cancer et les estimations de la population ont été regroupées selon le groupe d'âge (moins de 1 an [nourrissons], 1 à 4 ans, 5 à 9 ans et 10 à 14 ans), l'année du diagnostic, le sexe et la région au moment du diagnostic (Colombie-Britannique, provinces des Prairies [Alberta, Saskatchewan et Manitoba], Ontario, Québec, provinces de l'Atlantique [Nouveau-Brunswick, Île-du-Prince-Édouard, Nouvelle-Écosse et Terre-Neuve-et-Labrador] et territoires [Yukon, Territoires du NordOuest et Nunavut]). Ce regroupement a été effectué pour notre analyse car le nombre de cas de cancer était trop faible pour fournir des estimations stables de certains cancers à l'échelle de chaque province pour les Prairies, les provinces de l'Atlantique et les territoires. Les taux pour chaque catégorie ont été calculés en divisant le nombre de cas par catégorie par les effectifs de population correspondants. Les taux selon l'âge ont été normalisés sur la base de la population du Canada de 2011, au moyen de la méthode directe, pour obtenir des taux d'incidence normalisés selon l'âge (TINA) par million d'enfants.

Le Joinpoint Regression Program, un logiciel statistique d'analyse des tendances, a été utilisé pour déterminer les variations dans les tendances des TINA annuels de certains cancers de 1992 à 2010 ${ }^{14}$. La variable réponse était le logarithme naturel du TINA et la variable indépendante était l'année de diagnostic du cancer. Nous avons effectué des analyses distinctes en fonction du type de cancer, du sexe, de l'âge et de la région. Nous avons calculé la variation annuelle en pourcentage (VAP) des taux d'incidence du cancer en adaptant un modèle de régression linéaire par morceaux, en supposant un taux constant de variation du logarithme du TINA annuel dans chaque segment ${ }^{15}$. La pente estimée d'après ce modèle a ensuite été retransformée pour obtenir une augmentation ou une diminution annuelle en pourcentage du taux. Le test de la VAP est basé sur un test $t$ asymptotique. La VAP a été jugée statistiquement significative lorsque son intervalle de confiance (IC) à $95 \%$ ne comprenait pas zéro $(p<0,05)$. Les points de connexion des segments linéaires sont appelés «points de retournement » ou " points de jonction ». Les modèles rendent compte des erreurs types estimées des TINA. Afin de réduire la probabilité d'obtenir des variations des tendances qui n'en sont pas réellement, nous avons utilisé au moins cinq observations d'un point de jonction à la fin des données et au moins quatre observations entre les points de jonction. La signification statistique des variations de tendances (points de jonction) a été déterminée au moyen de tests de permutation de Monte Carlo avec correction de Bonferroni pour tenir compte de la probabilité de surajustement des multiples tests (le niveau de signification global était de 0,05).

Pour assurer la confidentialité et éviter toute possibilité de divulgation par recoupements, conformément aux exigences de déclaration du RCC, les chiffres sur l'incidence présentés dans les tableaux et à la figure 1 ont été arrondis de façon aléatoire à un multiple de 5. De ce fait, une fois les données regroupées, les totaux ne correspondent pas nécessairement à la somme des valeurs individuelles. Les TINA ont été calculés avec les chiffres réels. Les TINA et les VAP ne sont pas présentés lorsque les données arrondies correspondantes sont inférieures à 30. En outre, les classifications élargies des leucémies lymphoïdes, à l'exception de la leucémie lymphoblastique à précurseurs, ne sont pas présentées, car les cas dans ces sous-groupes initialement codés dans la CIM-O-2 ne contiennent pas les renseignements requis pour être convertis pour la $\mathrm{CIM}-\mathrm{O}-3^{10}$. De plus, les résultats par région ne sont fournis que pour les 12 grandes catégories diagnostiques et les sous-types dont les VAP sont significatives.

\section{Résultats}

Comme l'exhaustivité de la collecte de données sur les tumeurs bénignes du SNC était variable selon les provinces (données non illustrées), ce qui est susceptible d'avoir des répercussions sur les comparaisons dans le temps et d'une région à l'autre (voir la section Analyse), les résultats décrits dans cette section portant sur tous les cancers confondus et les tumeurs du 
FIGURE 1

Distribution des nouveaux cas de cancer diagnostiqués chez les enfants de moins de 15 ans selon le sexe et les groupes d'âge au Canada, 2006-2010
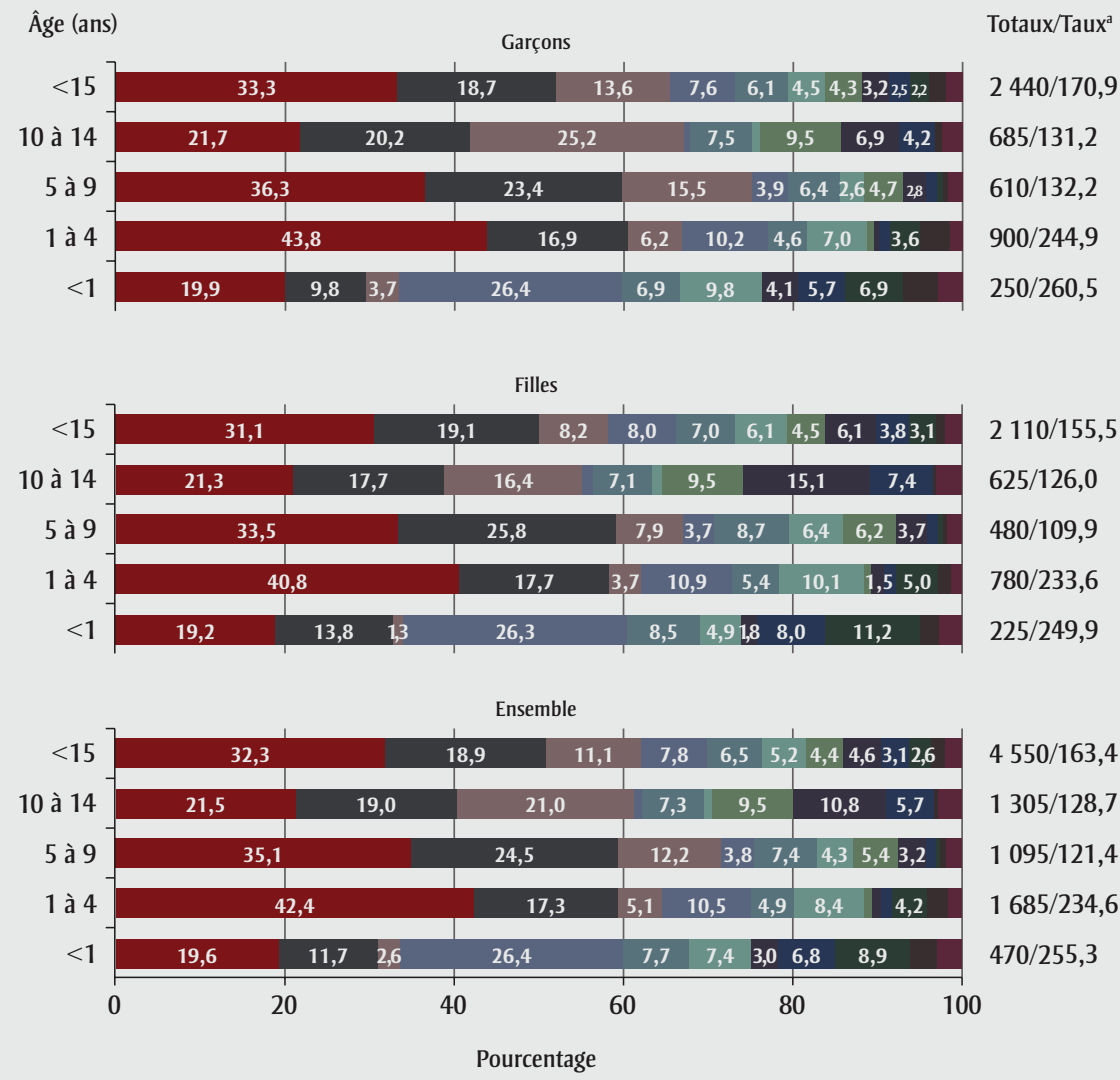

$\begin{array}{llll}\square \text { Leucémies } & \text { Tumeurs du SNC } & \text { Lymphomes } & \text { Neuroblastome } \\ \text { Sarcomes des tissus mous } & \text { Tumeurs rénales } & \text { Os } & \text { Carcinomes } \\ \text { Cellules germinales } & \square \text { Rétinoblastome } & \text { Tumeurs hépatiques } & \text { Tutres }\end{array}$

Sources des données : Base de données du Registre canadien du cancer (RCC) à Statistique Canada et Registre du cancer du Québec (2008-2010).

Remarque : Les taux ont été normalisés selon la population canadienne de 2011 pour tous les âges combinés.

a Le nombre de nouveaux cas a été arrondi de façon aléatoire, à l'unité inférieure ou supérieure, selon un multiple de 5.

SNC ne sont fondés que sur les tumeurs malignes alors que les résultats des modèles de régression Joinpoint les mieux ajustés présentés dans les tableaux 1 à 5 concernant ces deux catégories englobent les tumeurs bénignes du SNC.

\section{Nombre de cas et taux d'incidence récents (2006 à 2010)}

La figure 1 présente la répartition des cancers primitifs au Canada entre 2006 et 2010 en fonction du groupe d'âge pour les garçons et les filles, combinés et séparément. $\mathrm{Au}$ cours de cette période, en moyenne 910 nouveaux cas ont été diagnostiqués chaque année chez les enfants de 14 ans et moins au Canada, soit 4550 nouveaux cas au total : 2440 (53,6 \%) chez les garçons et $2110(46,4 \%)$ chez les filles, ce qui équivaut à un ratio garçons:filles de 1,2:1. Le TINA annuel moyen était de 163,4 pour 1 million d'enfants, les garçons affichant un taux supérieur à celui des filles (170,9 contre 155,5 pour $10^{6}$ enfants). On a mesuré les TINA annuels moyens pour tous les cancers confondus en 2006-2010 les plus faibles dans les Prairies $(149,4$ pour $10^{6}$ ) et les plus élevés en Ontario $\left(170,1\right.$ pour $10^{6}$ ) (figure 2 ).

Alors que la plupart des cancers chez les adultes sont des carcinomes, les cancers de l'enfant présentent une grande diversité histologique et biologique et ne sont généralement pas d'origine épithéliale. Dans l'ensemble, les cancers le plus couramment diagnostiqués chez les enfants entre 2006 et 2010 ont été les leucémies (32,3\%), les tumeurs du SNC $(18,9 \%)$ et les lymphomes $(11,1 \%)$ (figure 1$)$, suivis du neuroblastome $(7,8 \%)$, du sarcome des tissus mous $(6,5 \%)$ et des tumeurs rénales $(5,2 \%)$. Les cinq types de cancer les plus courants étaient répartis de façon similaire dans chaque région, mais nous avons observé quelques variations dans les proportions et le classement dans la région de l'Atlantique (figure 2), sans doute en raison d'une erreur de type I attribuable aux faibles effectifs de population de la région. La répartition des cancers de l'enfant les plus fréquents était généralement équivalente chez les garçons et les filles, sauf pour les lymphomes, plus fréquents chez les garçons $(13,6 \%$ contre $8,2 \%)$, et les carcinomes (en particulier le cancer de la thyroïde), plus fréquents chez les filles $(6,1 \%$ contre $3,2 \%)$ (figure 1 ).

Environ la moitié des cas de cancer chez les enfants $(47,4 \%)$ ont été diagnostiqués chez les enfants de moins de 5 ans (figure 1). Les taux d'incidence selon l'âge chez les enfants de moins de 5 ans étaient environ deux fois plus élevés que ceux des enfants plus âgés. L'incidence la plus élevée a été observée chez les nourrissons de moins d'un an, accompagnée en général d'une diminution avec l'âge. Les cancers diagnostiqués variaient considérablement en fonction $\mathrm{du}$ groupe d'âge. Chez les nourrissons, les neuroblastomes étaient les cancers les plus courants et constituaient près du tiers de tous les cas $(26,4 \%)$, suivis des leucémies $(19,6 \%)$ et des tumeurs du SNC (11,7 \%). Les tumeurs embryonnaires de neuroblastomes, de rétinoblastomes et de néphroblastomes constituaient conjointement $42,6 \%$ de tous les diagnostics chez les nourrissons. Les leucémies dominaient chez les 1 à 4 ans, constituant $42,4 \%$ de tous les diagnostics, tandis que, chez les enfants de 5 à 9 ans et de 10 à 14 ans, les lymphomes et les tumeurs osseuses devenaient plus courants (lymphomes : respectivement $12,2 \%$ et $21,0 \%$, cancer des os : $5,4 \%$ et 9,5\%). De même, chez les enfants de 10 à 14 ans, les leucémies $(21,5 \%)$ et les tumeurs du SNC (19,0 \%) prédominaient.

\section{Tendances temporelles globales (1992 à 2010)}

Les tendances variaient beaucoup en fonction du type de cancer, sachant que le petit 


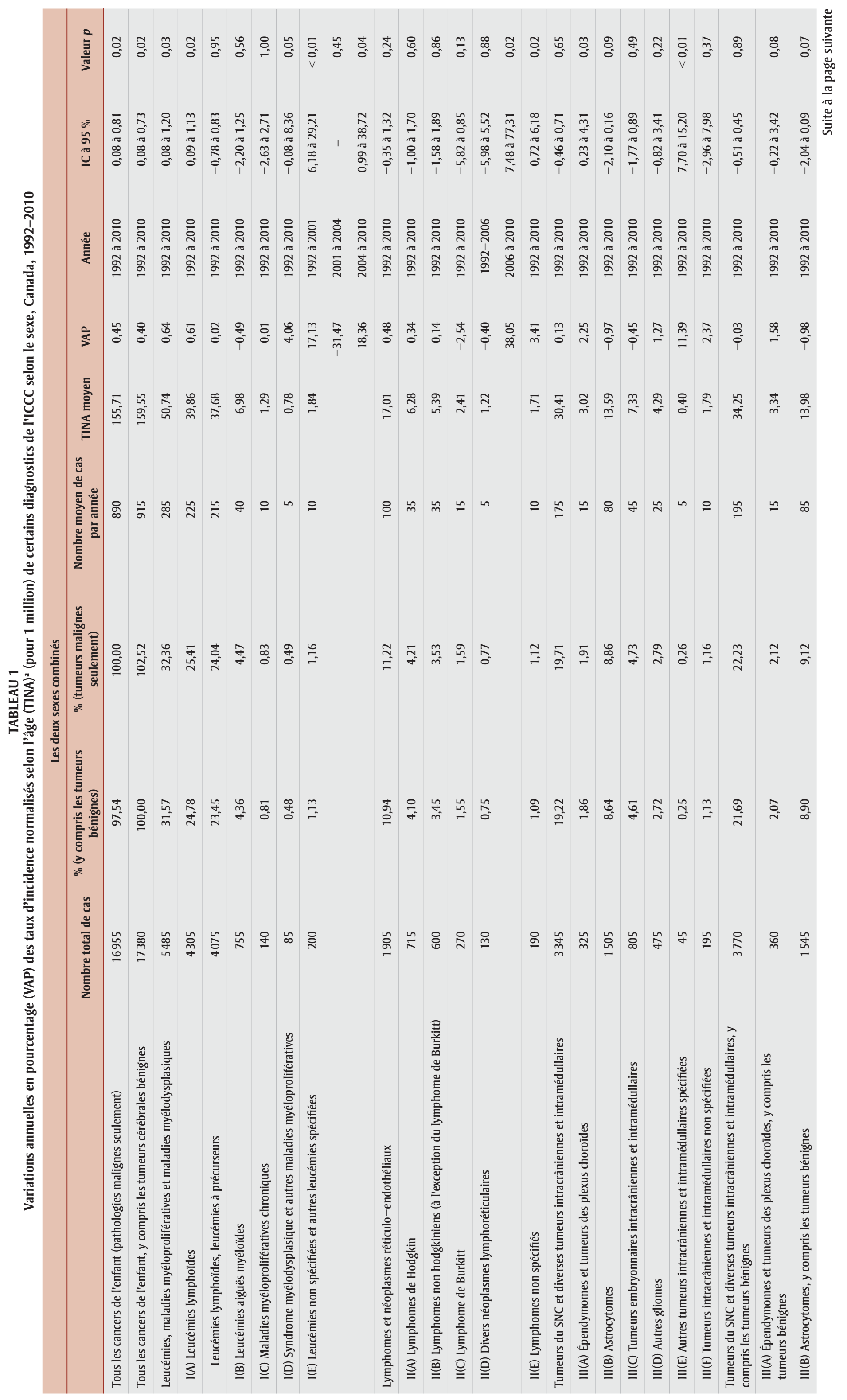




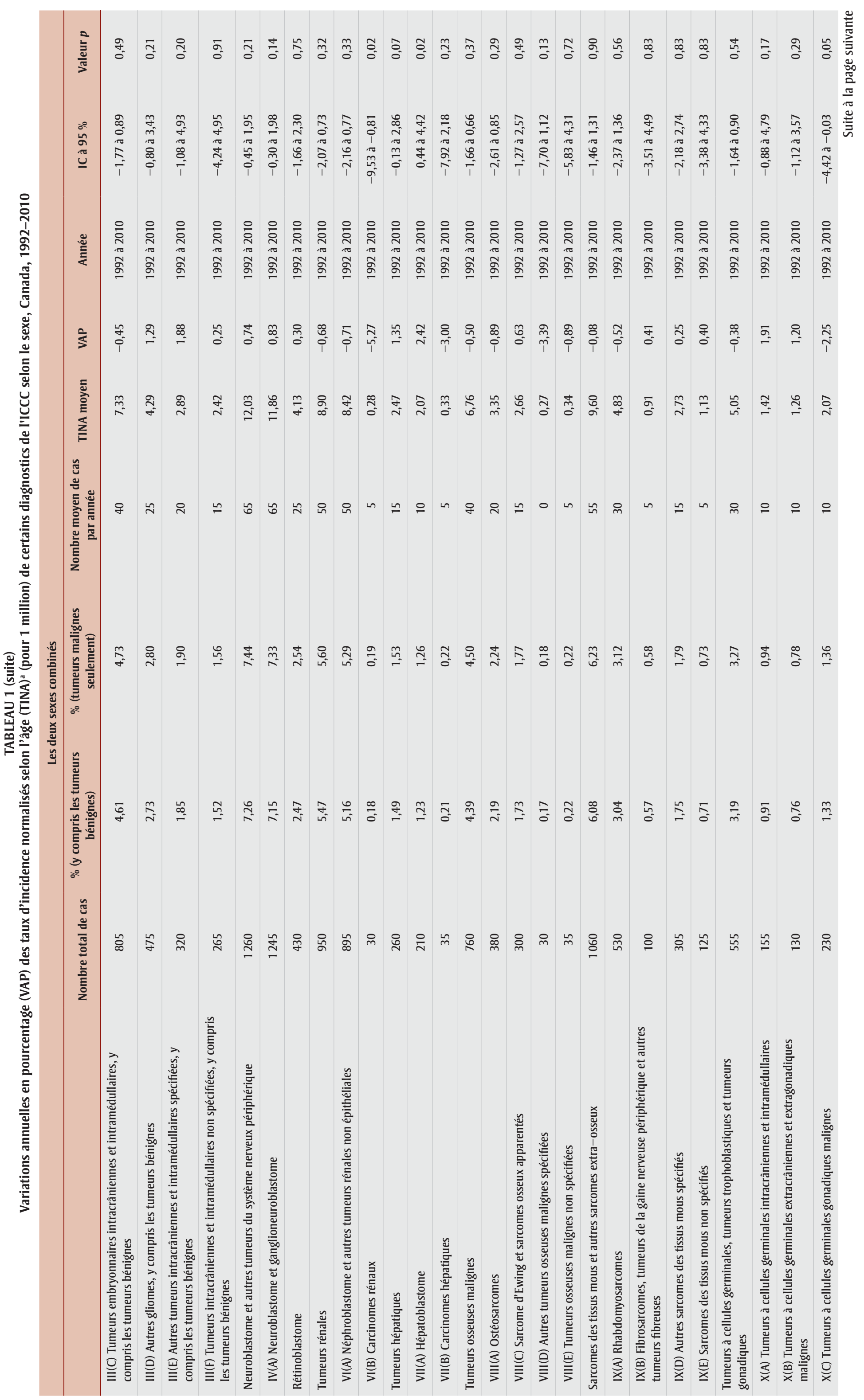




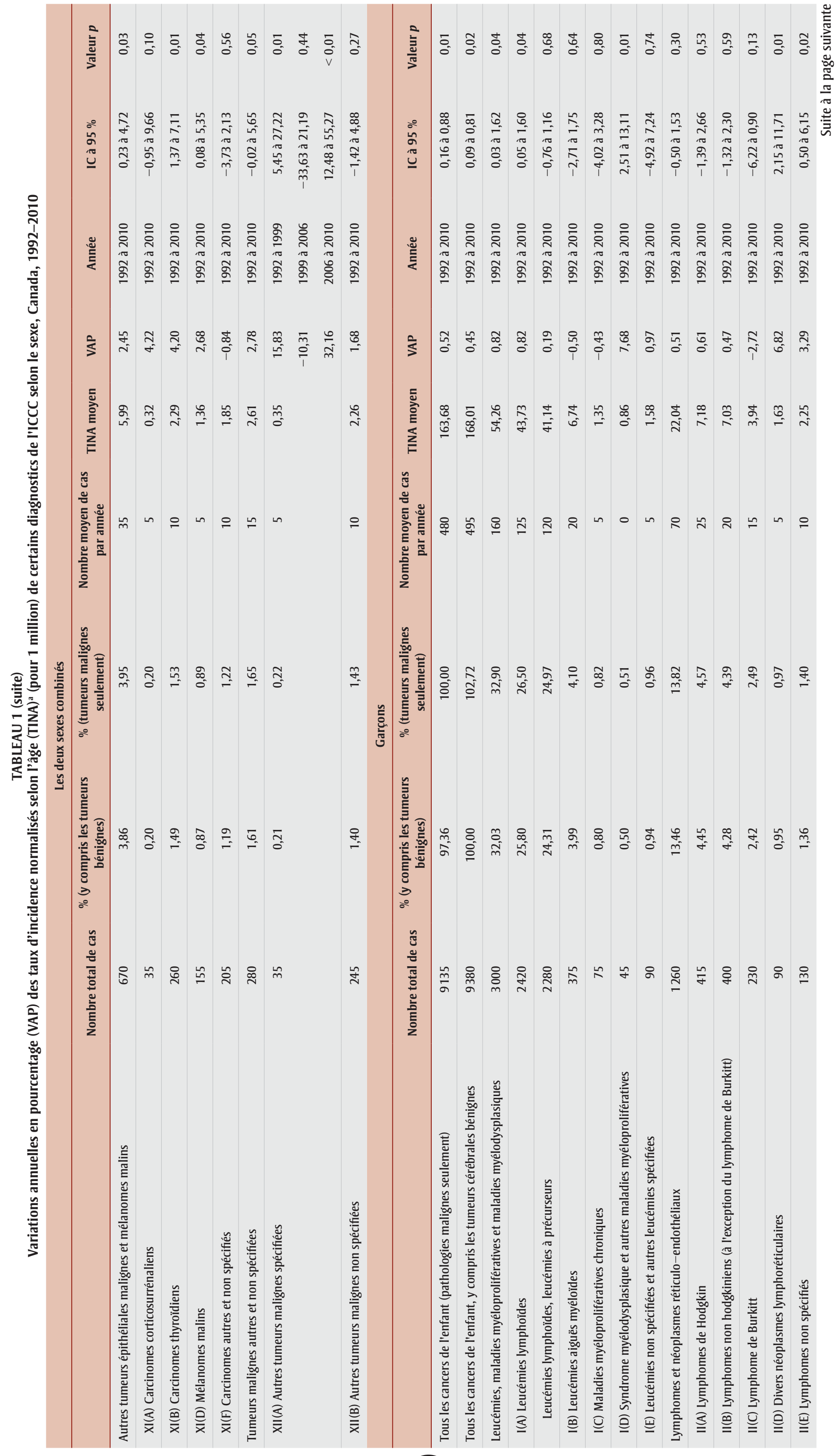




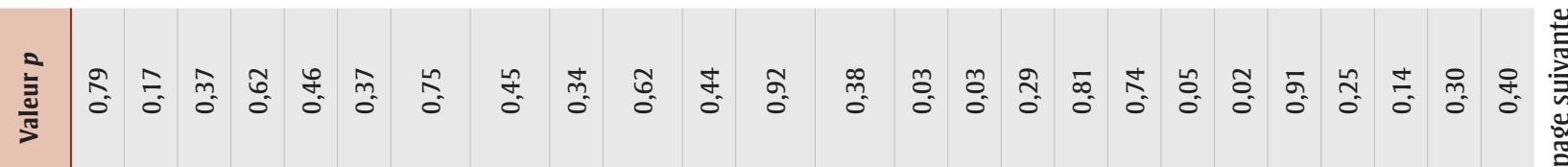

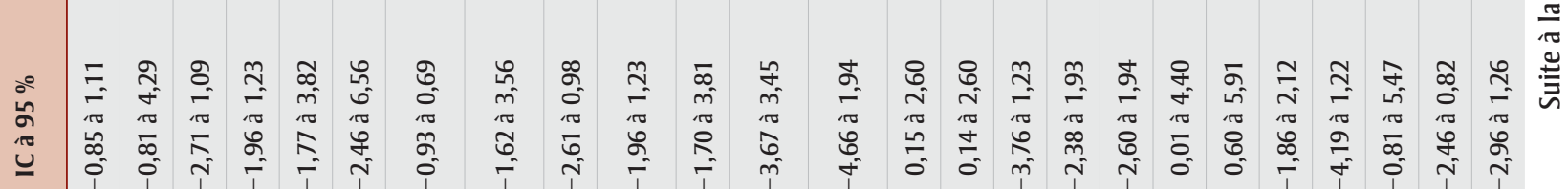

웅

产

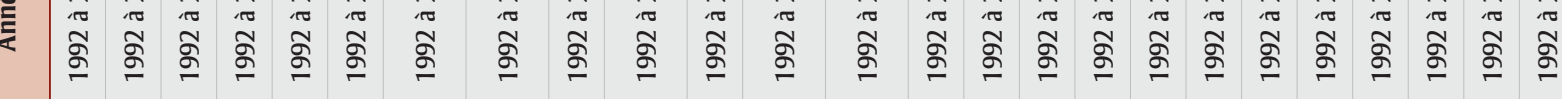

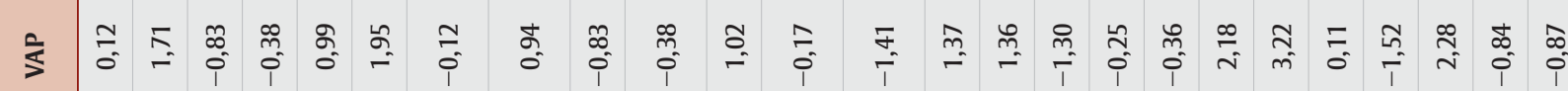

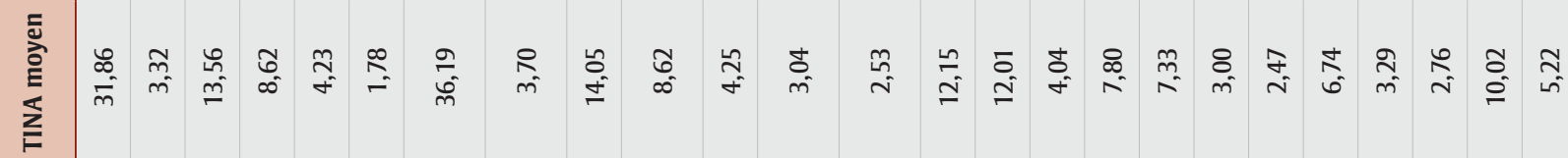
ป

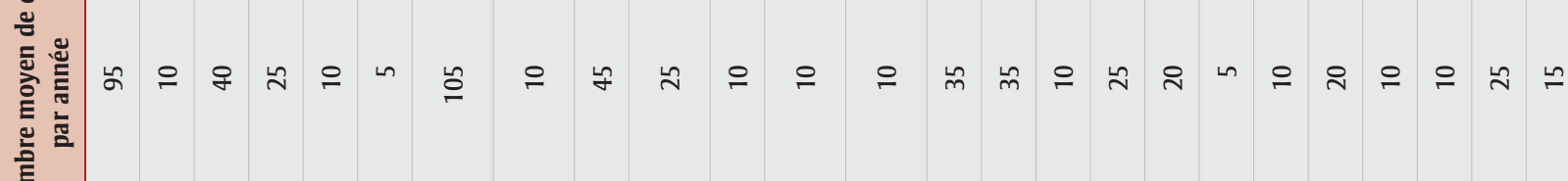
言

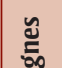

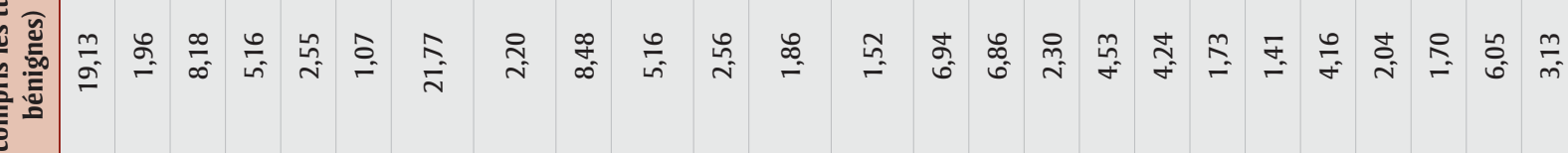

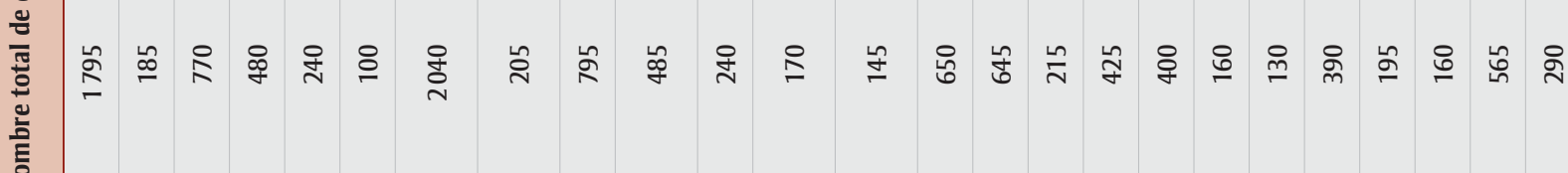

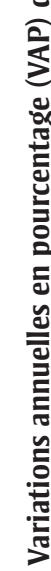

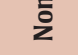

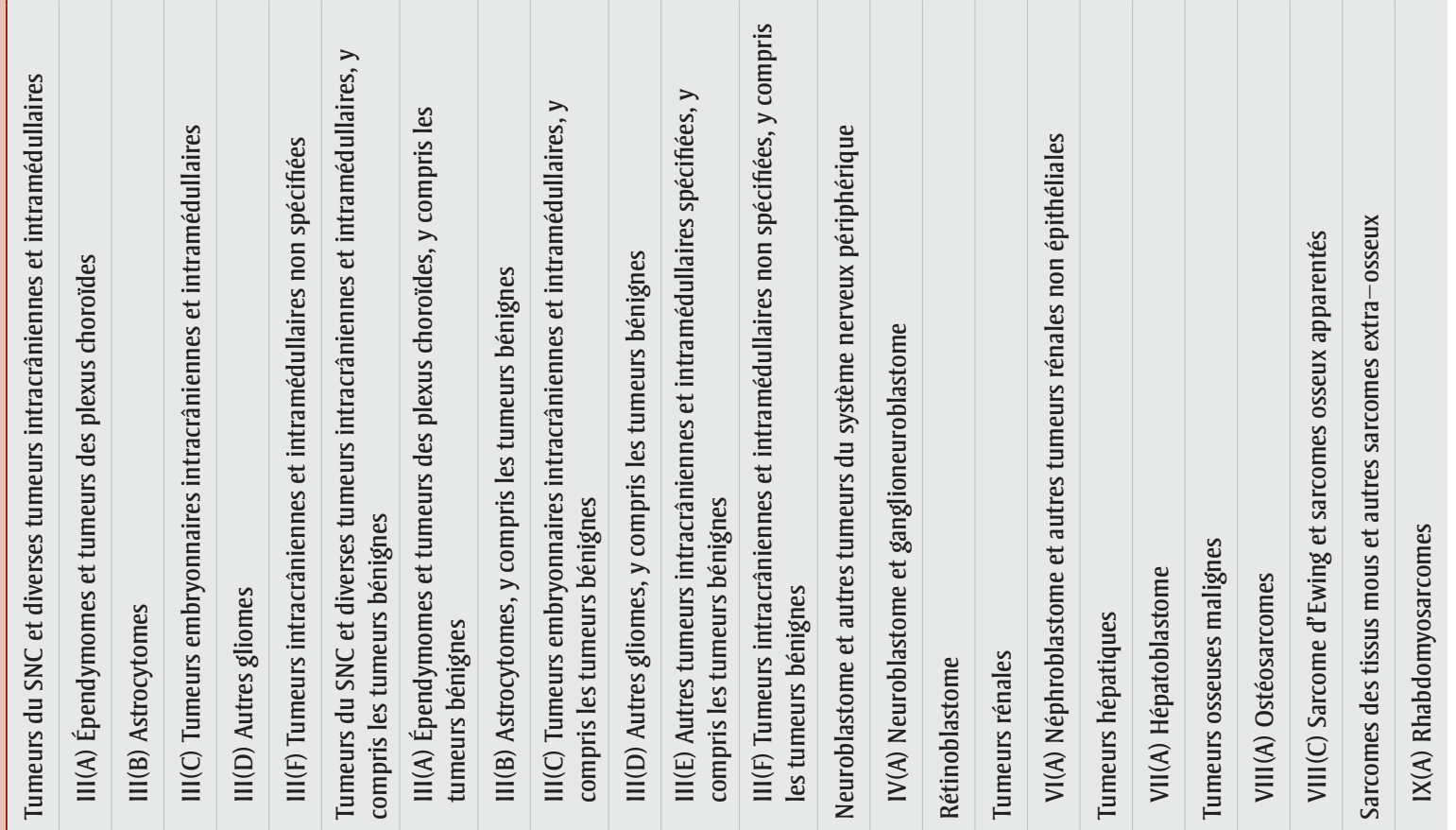




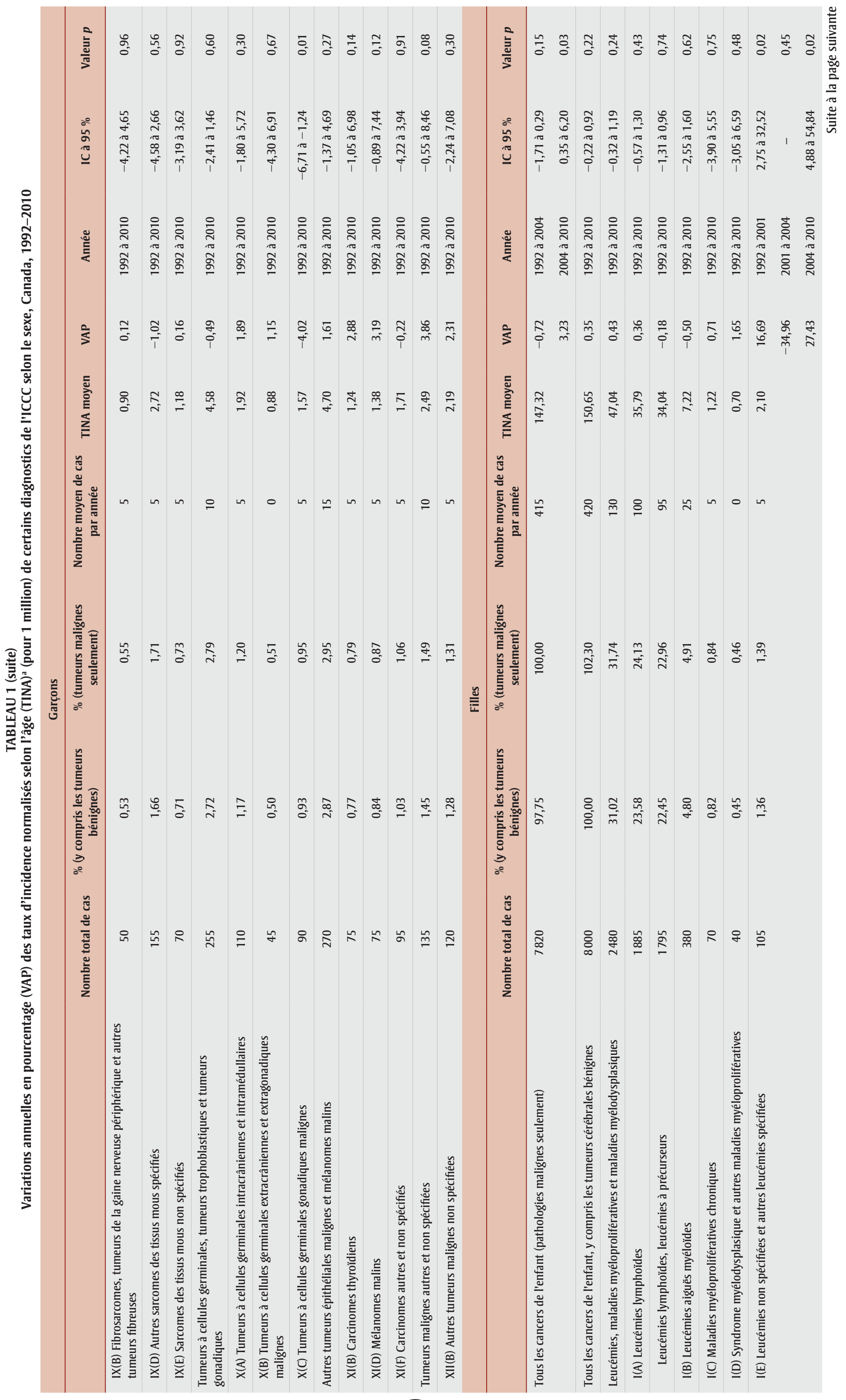




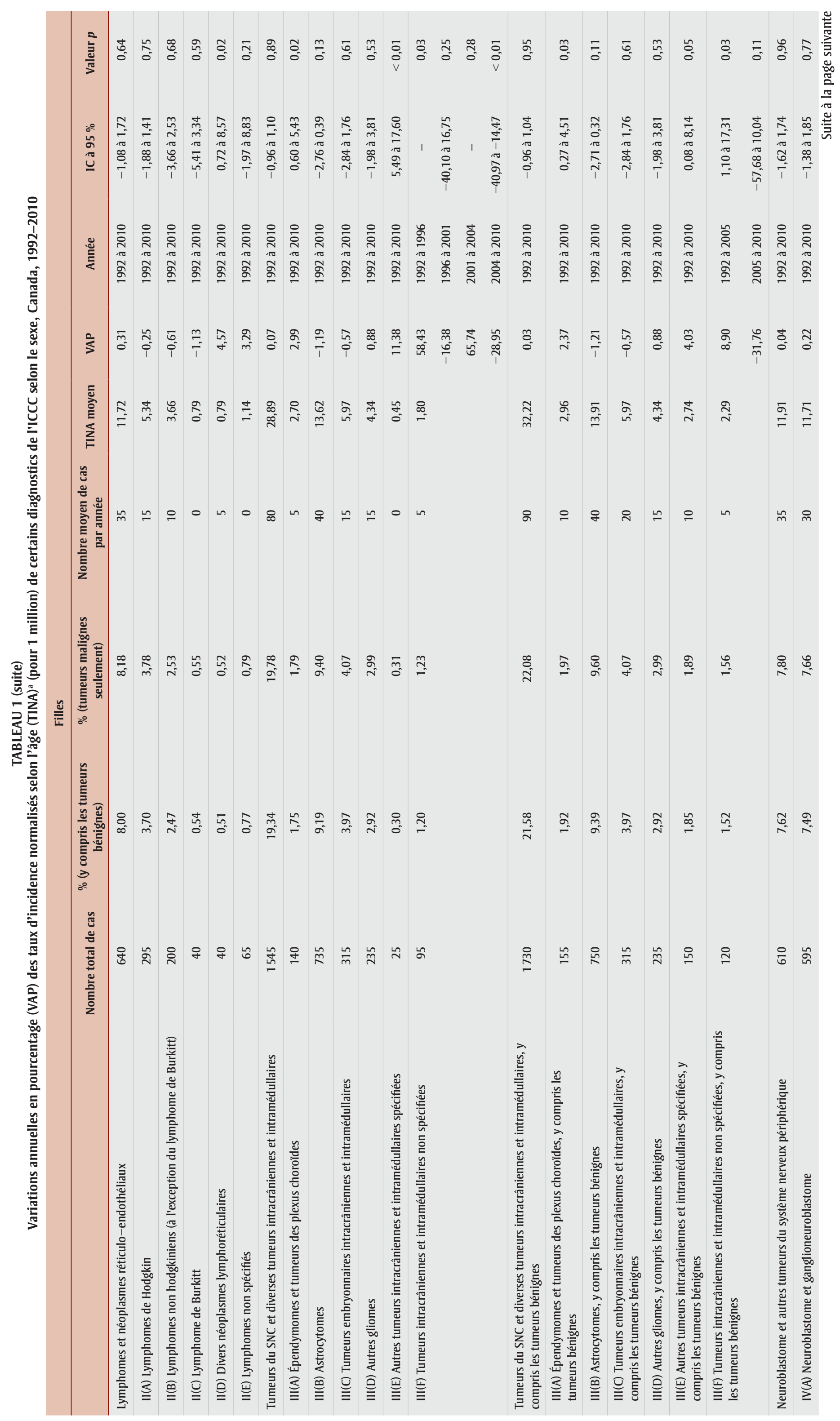




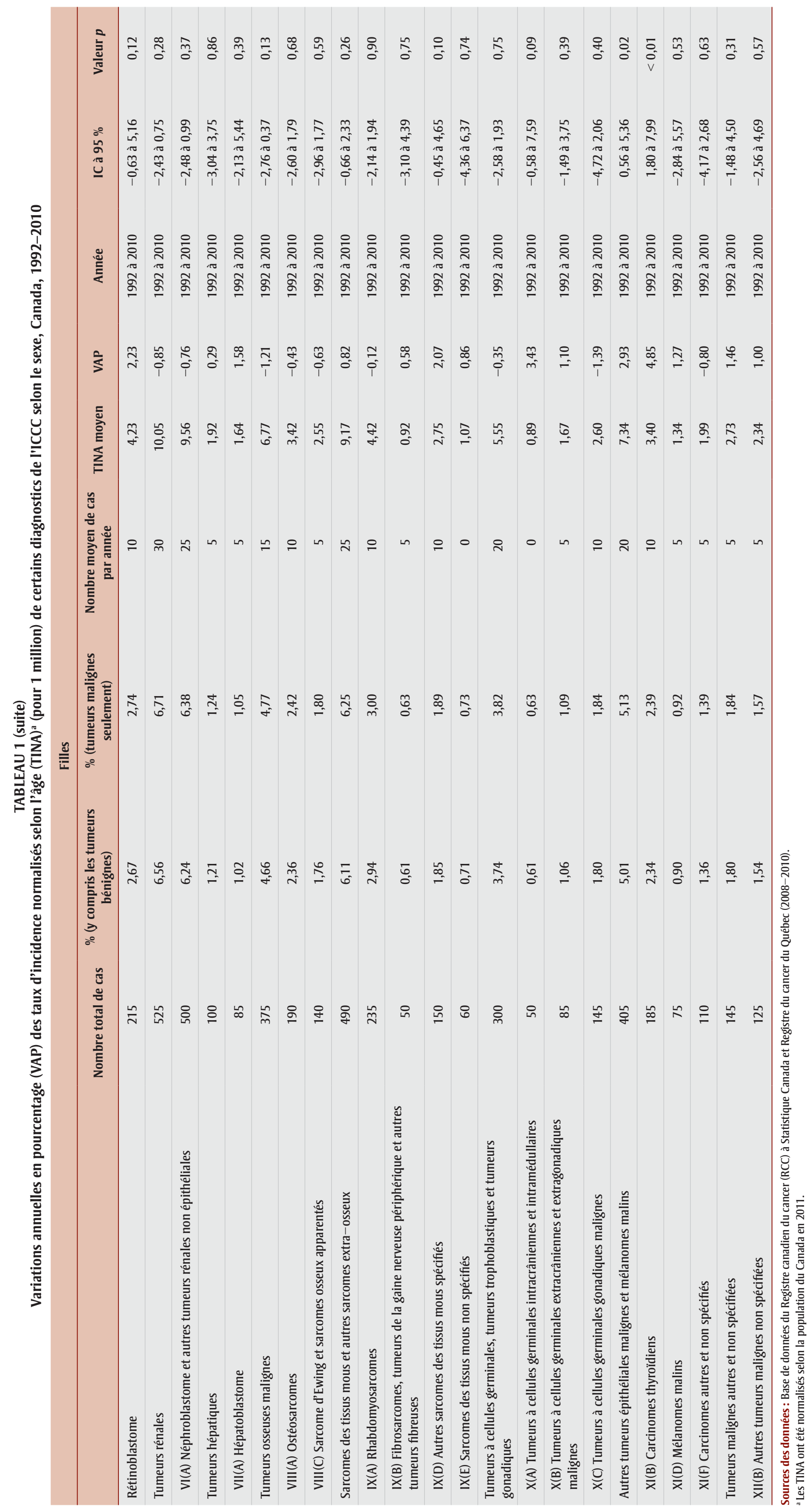




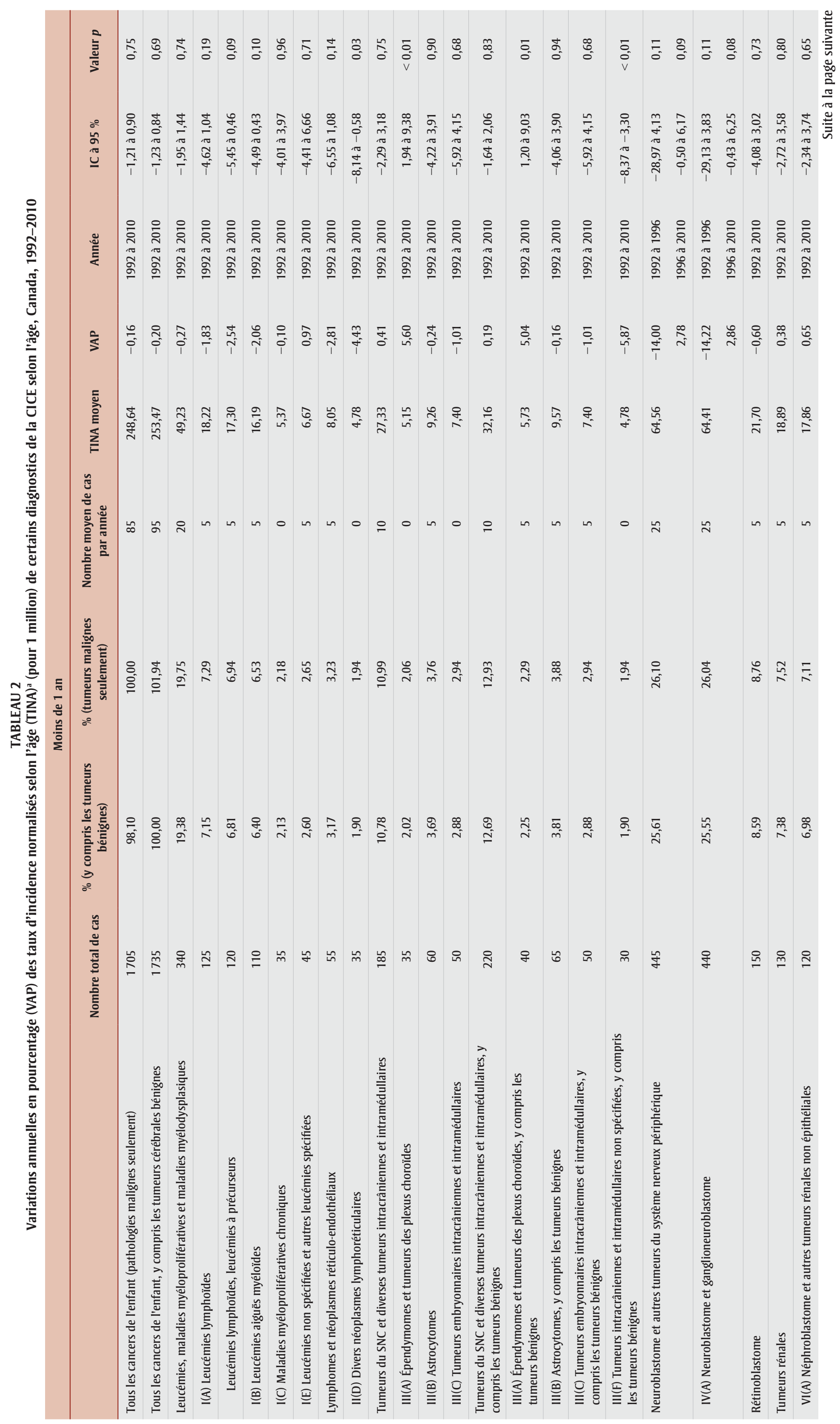




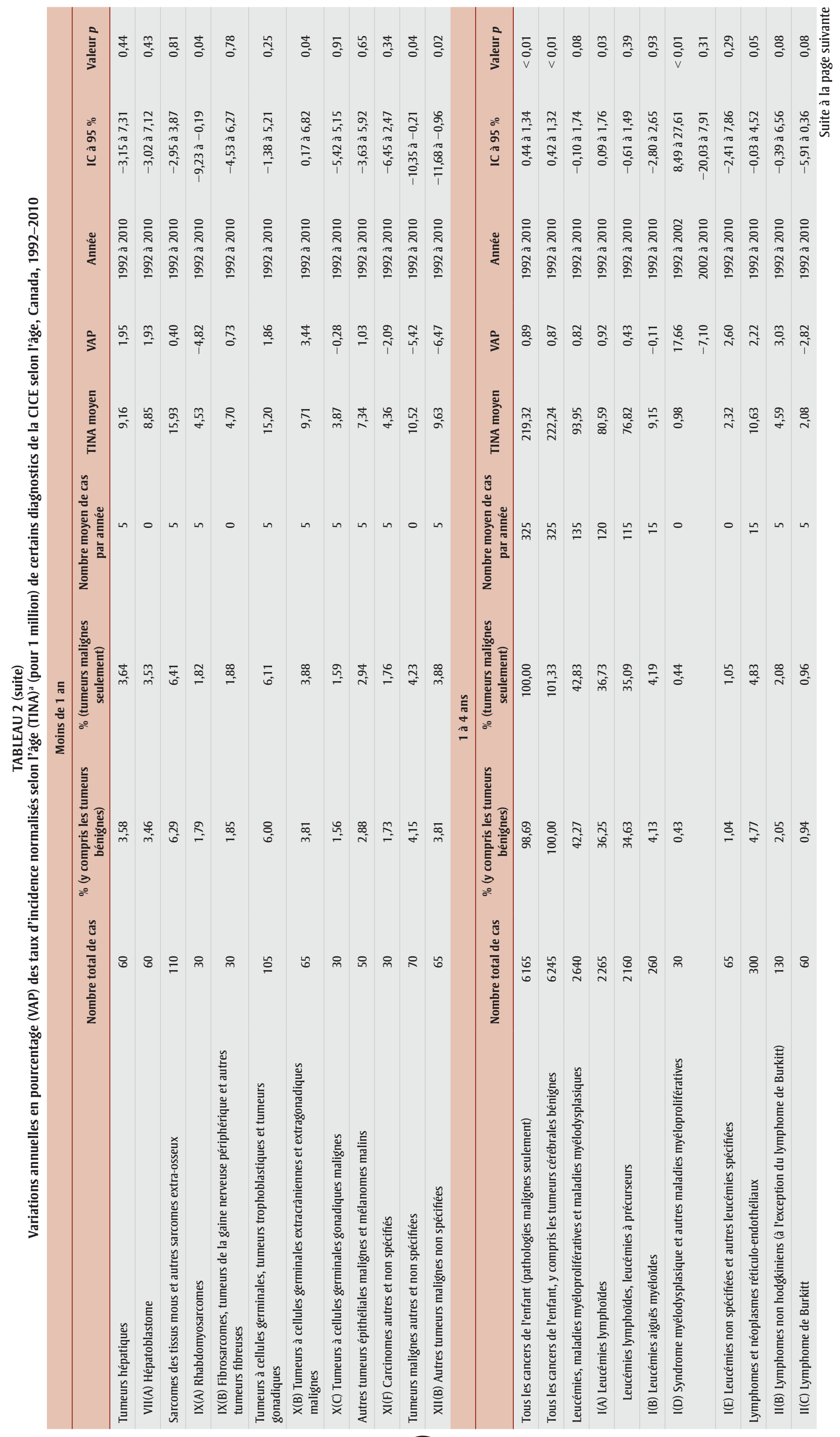




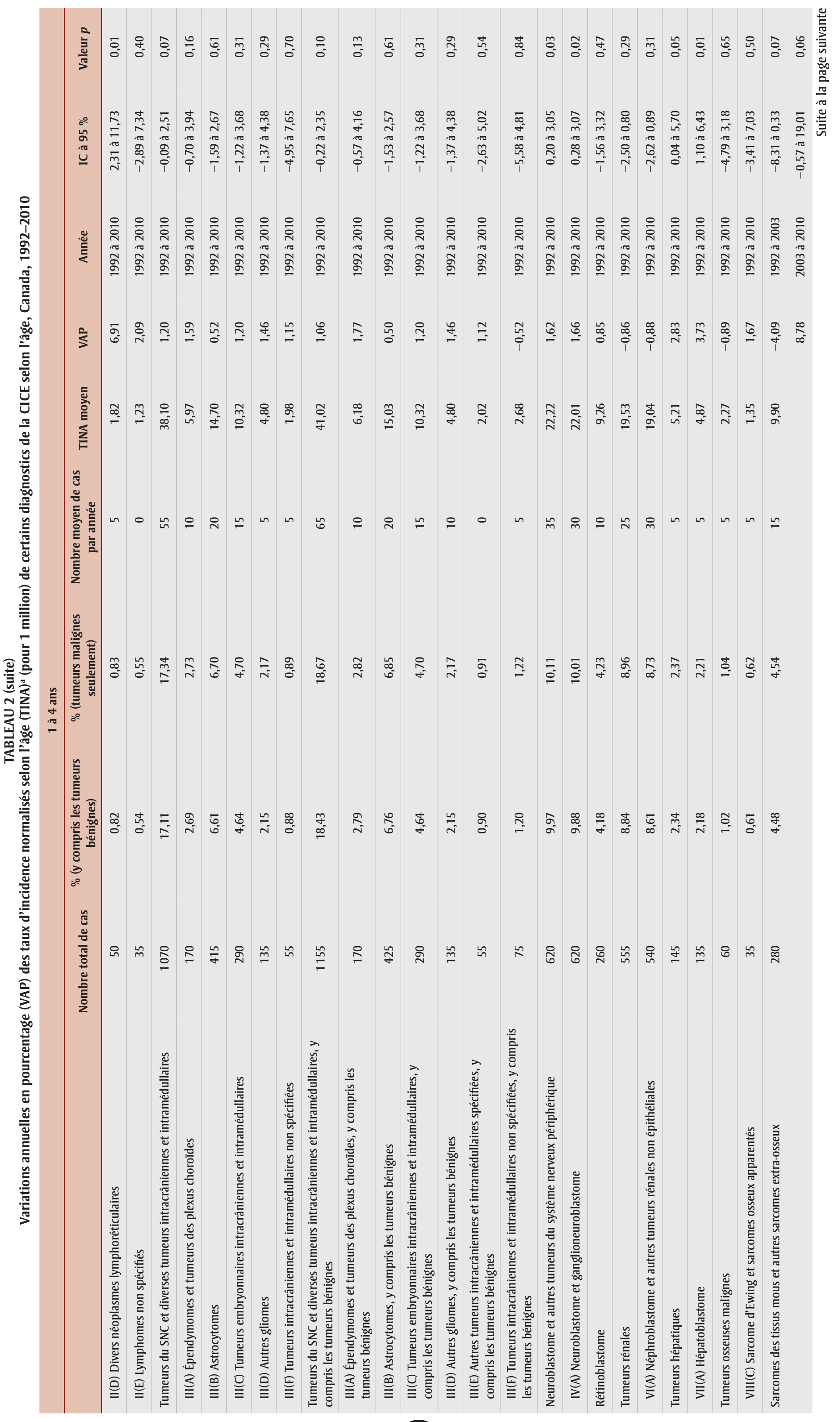




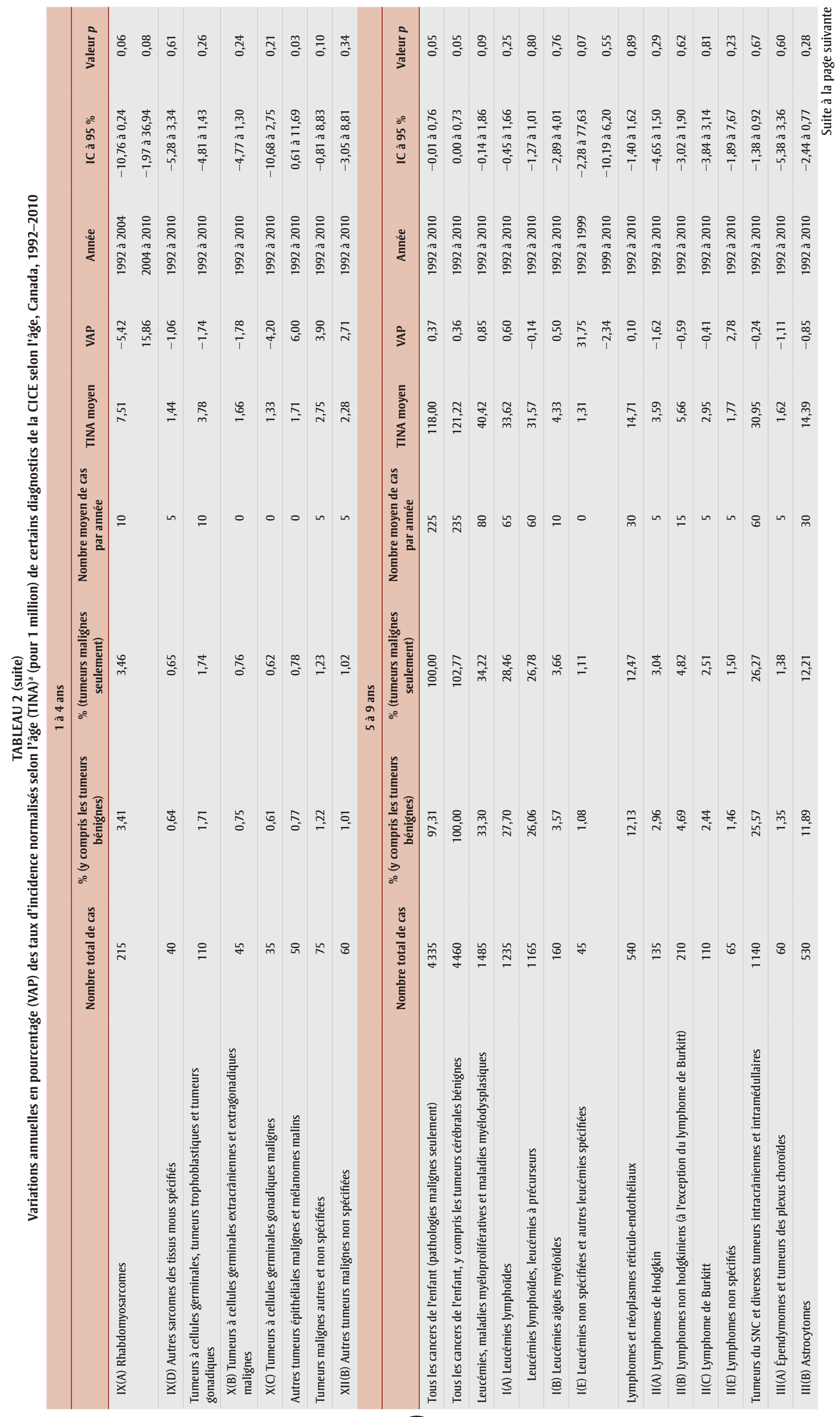




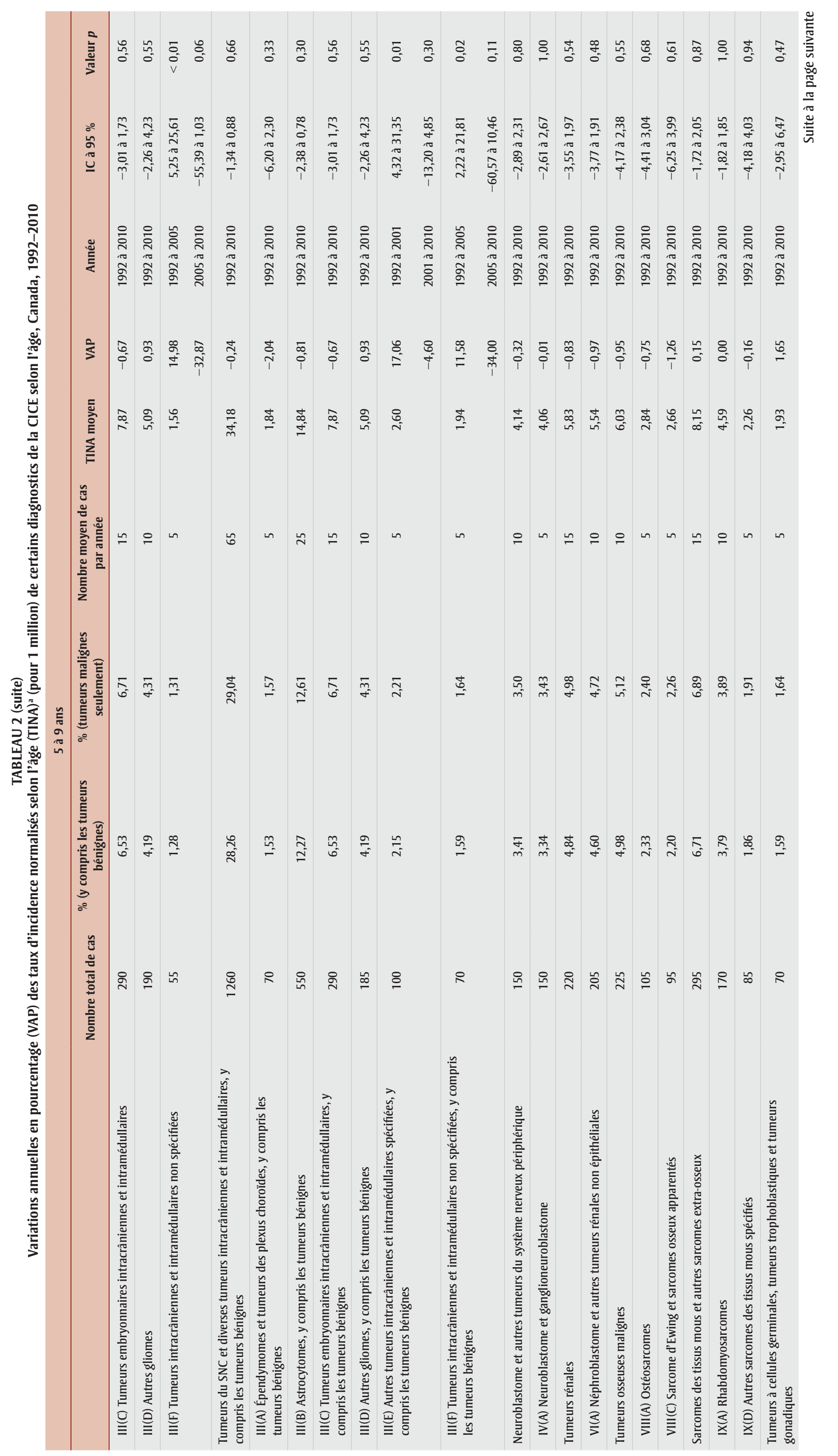




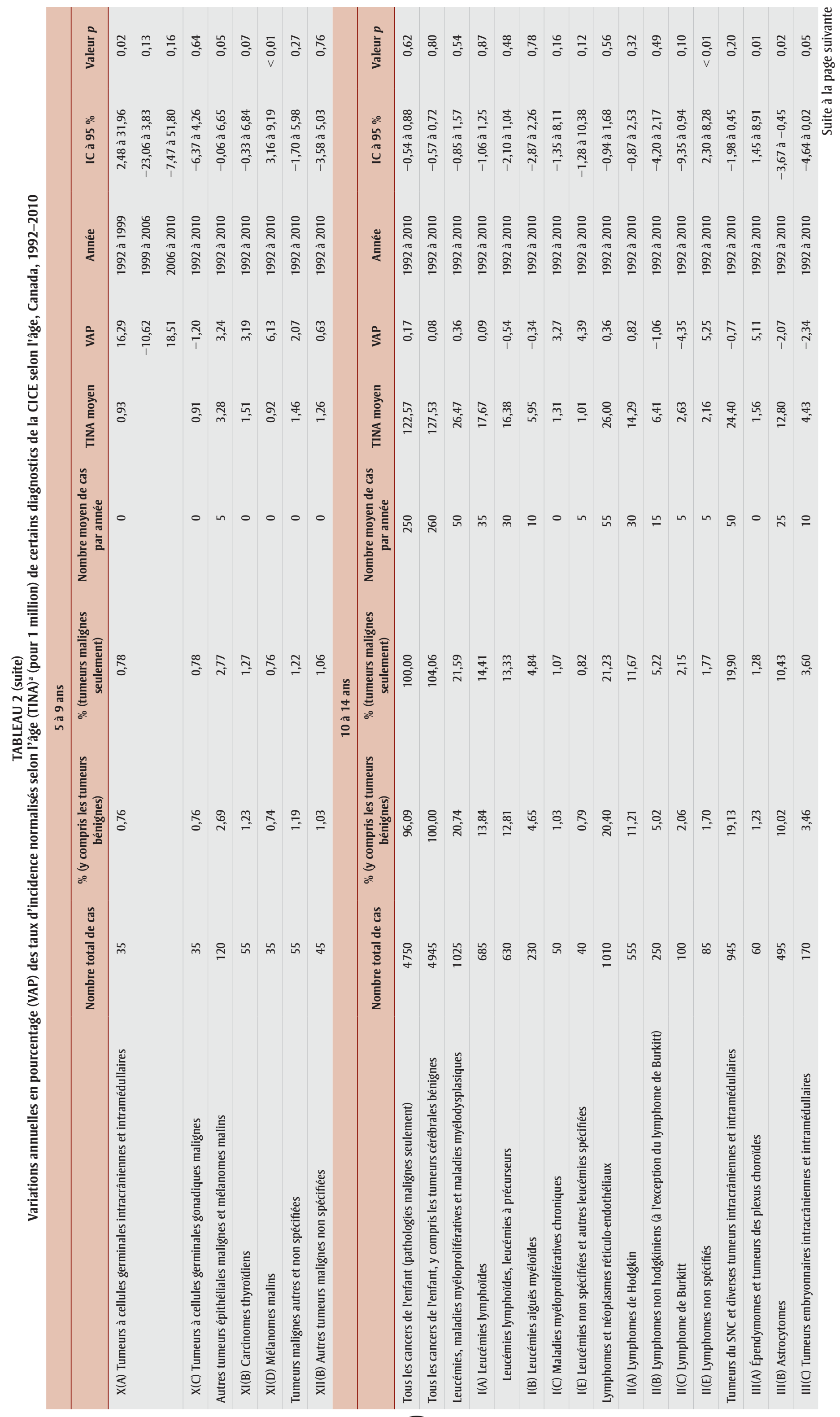




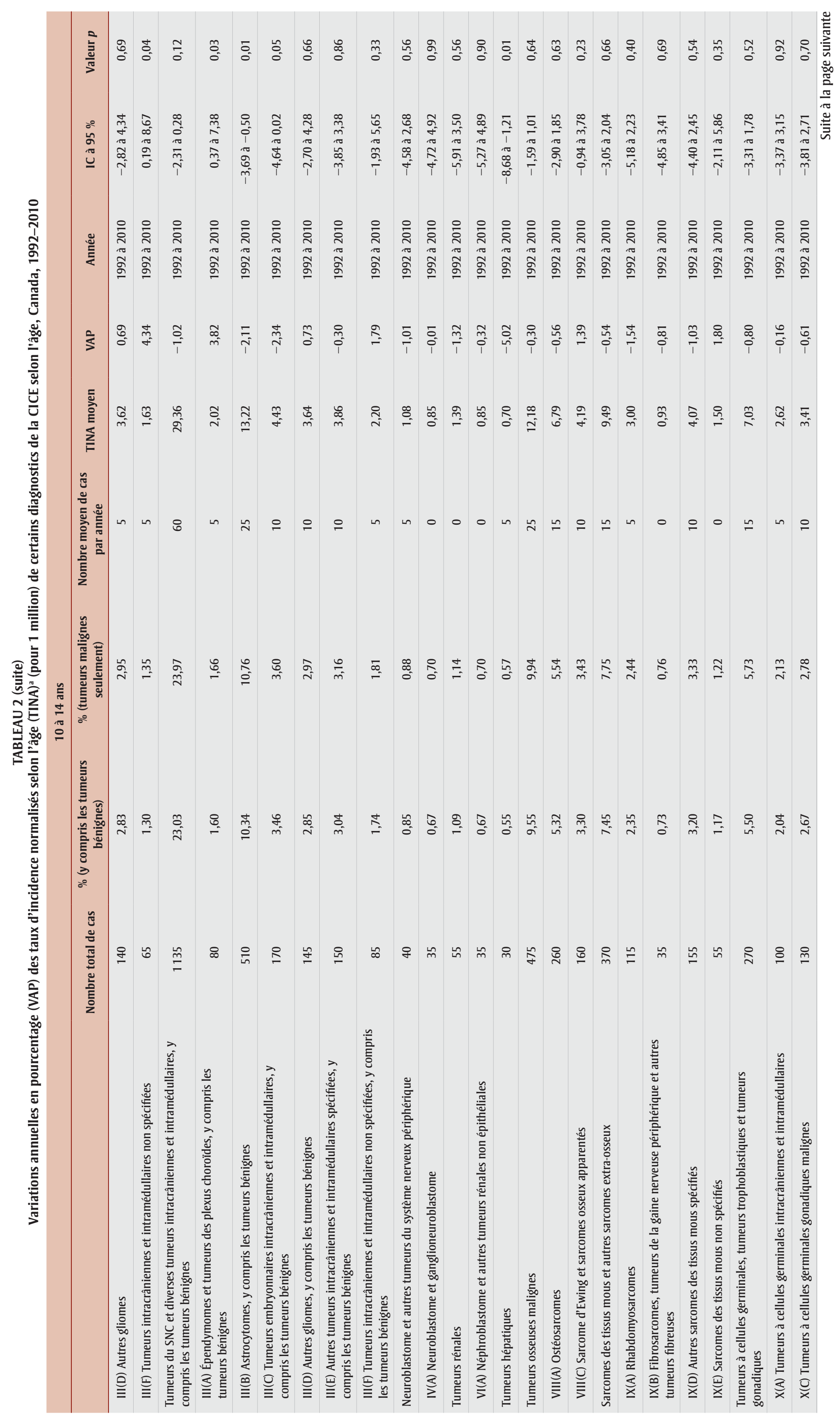




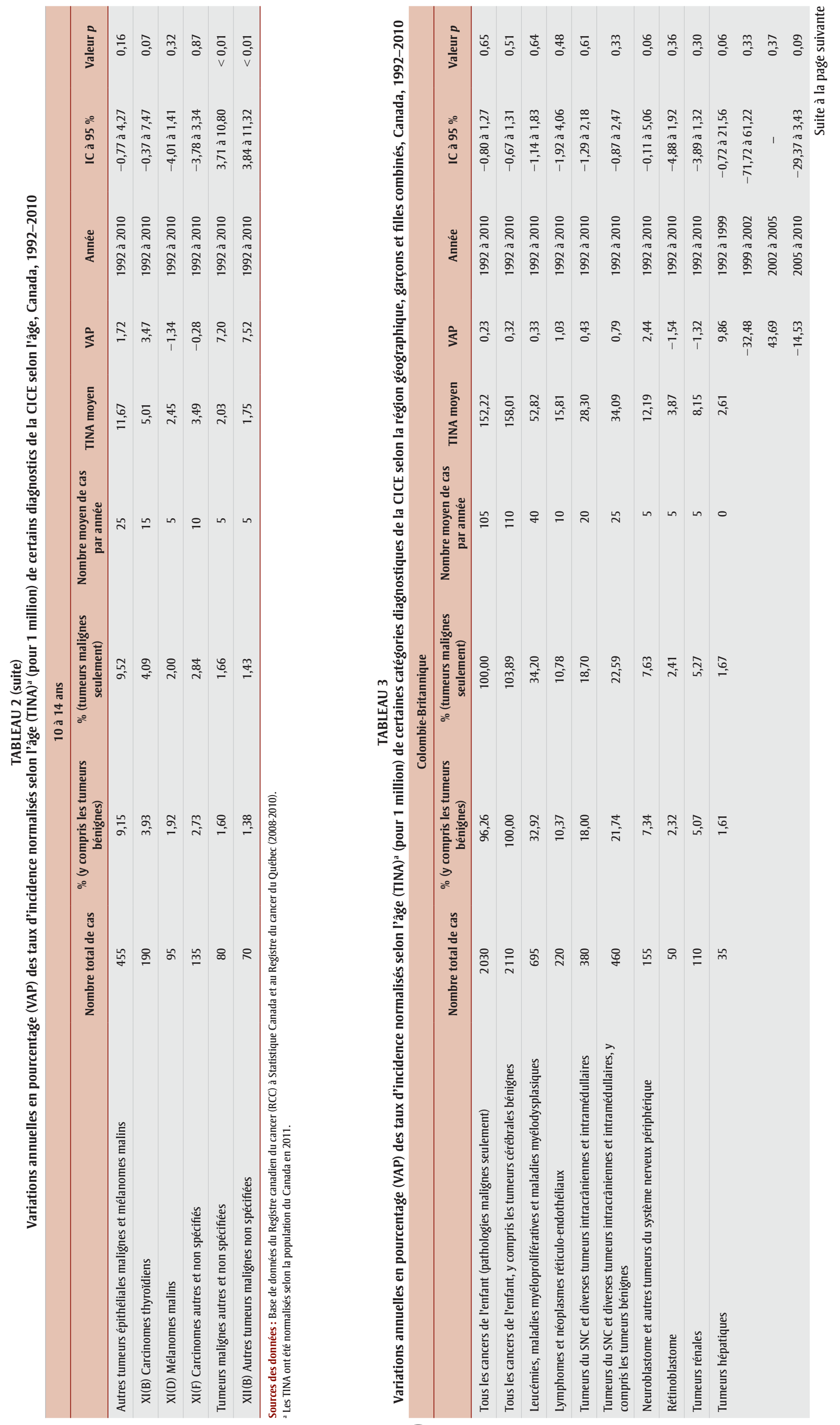




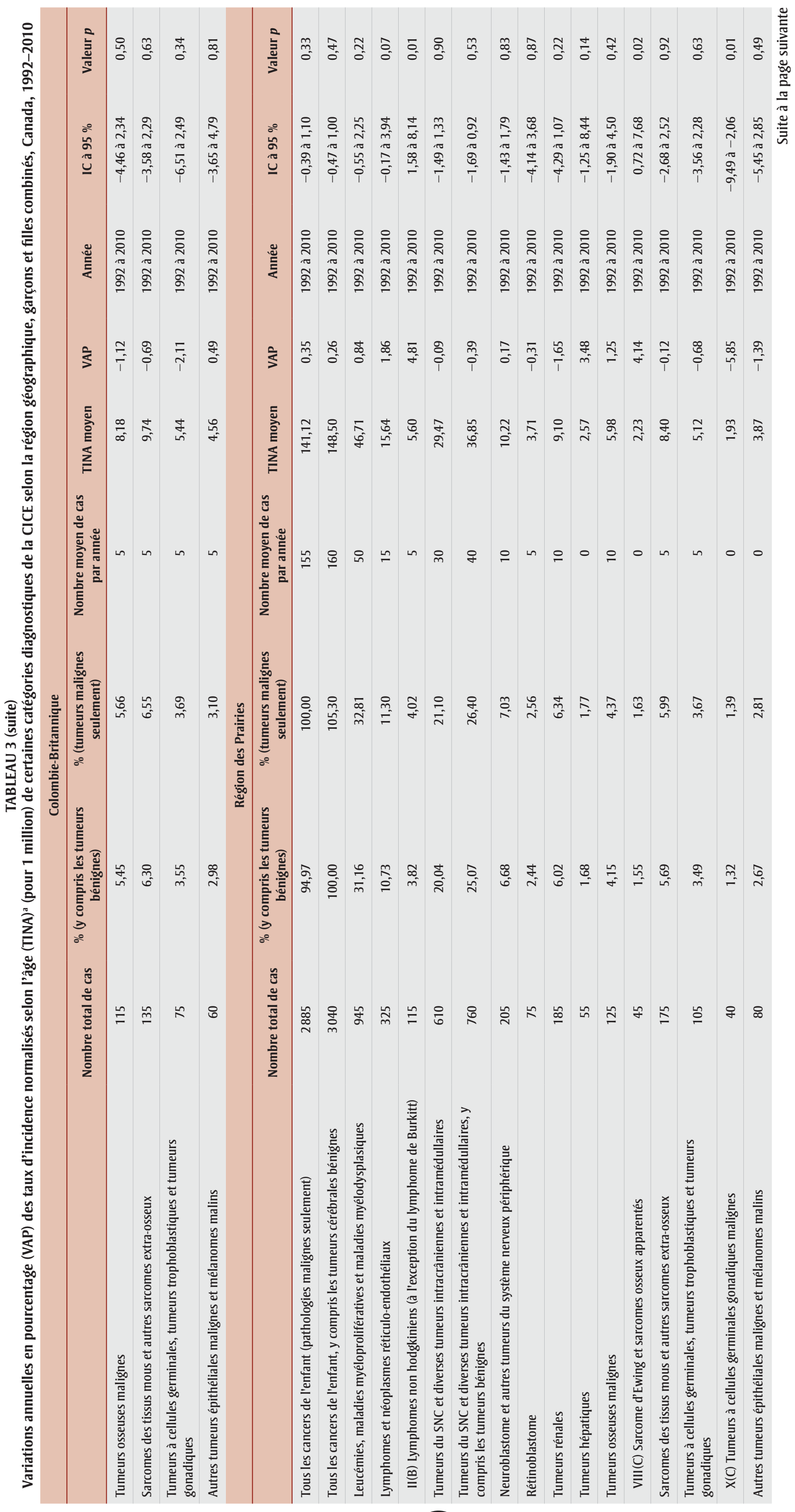




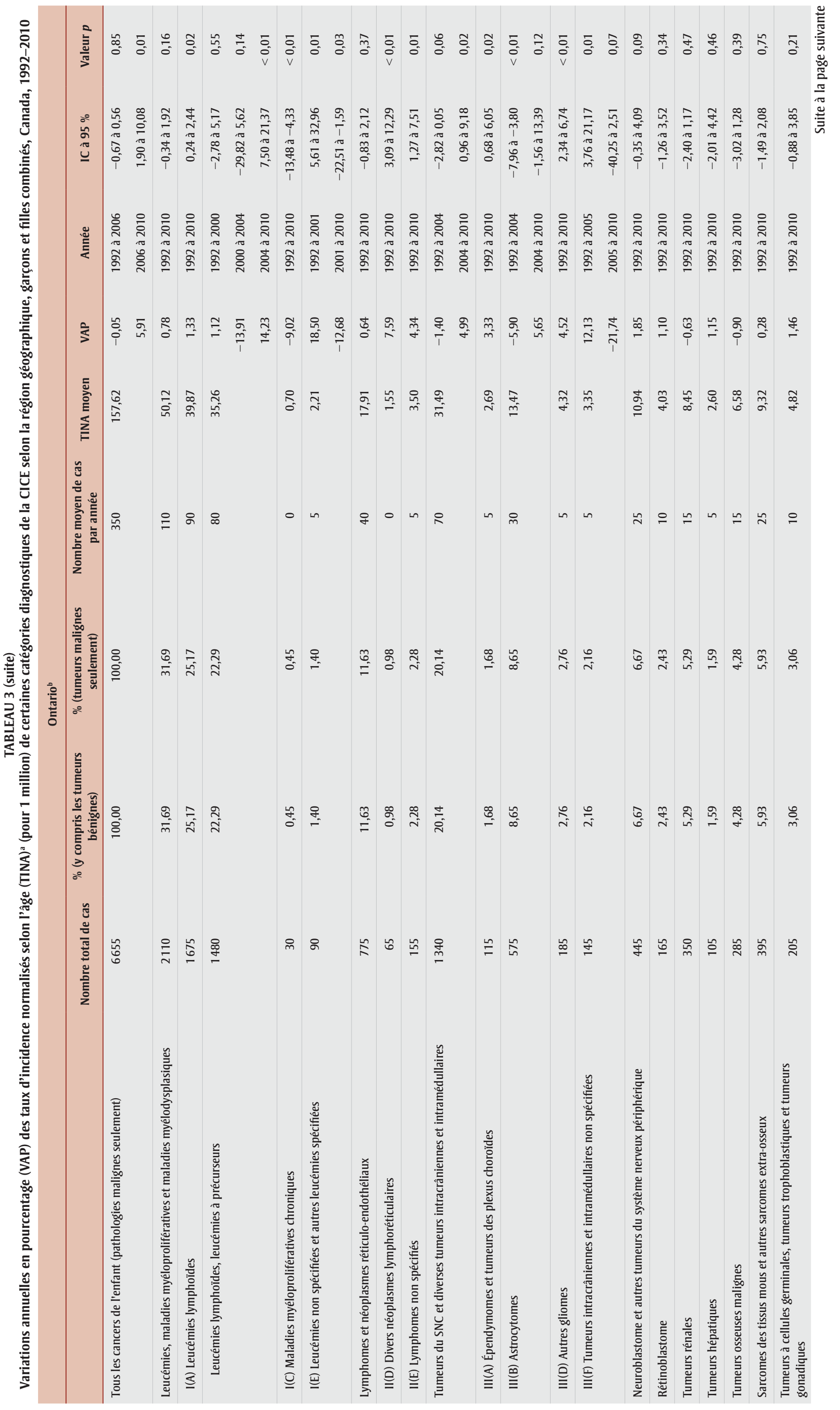




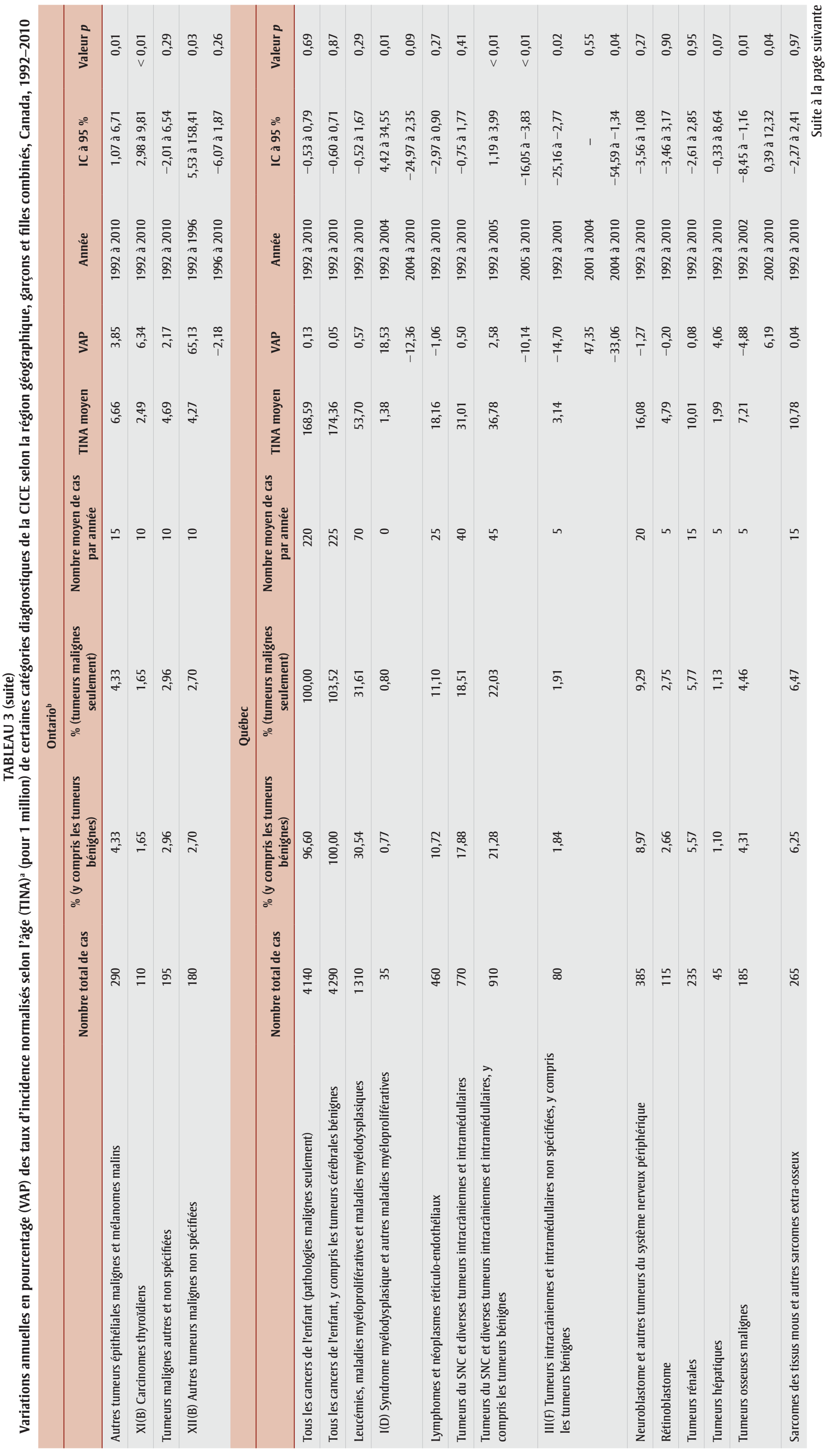




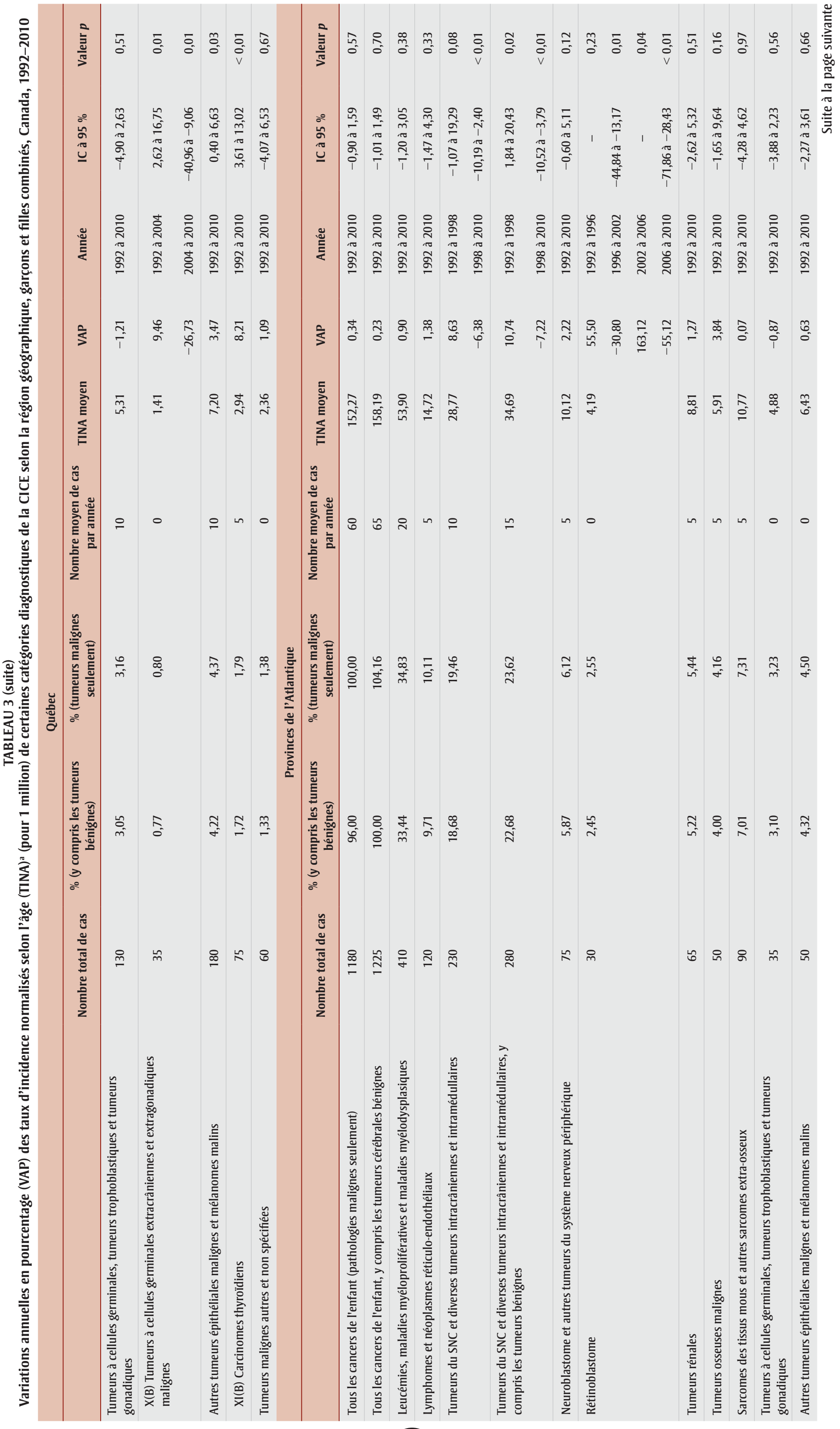




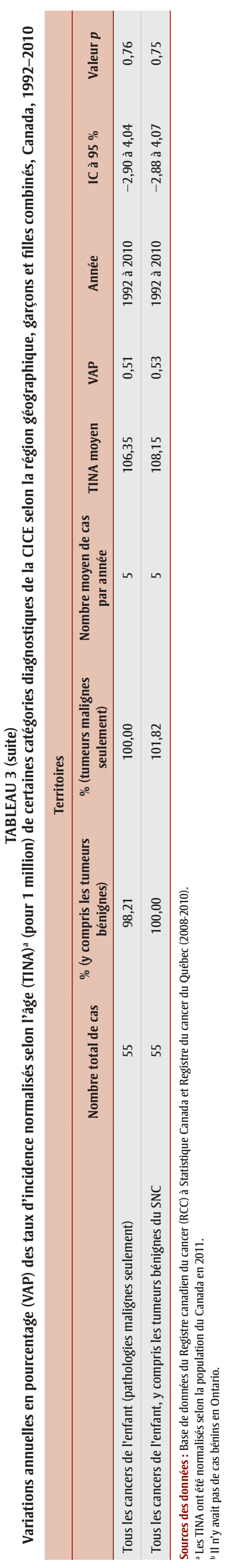

Vol 38, $n^{\circ} 3$, mars 2018

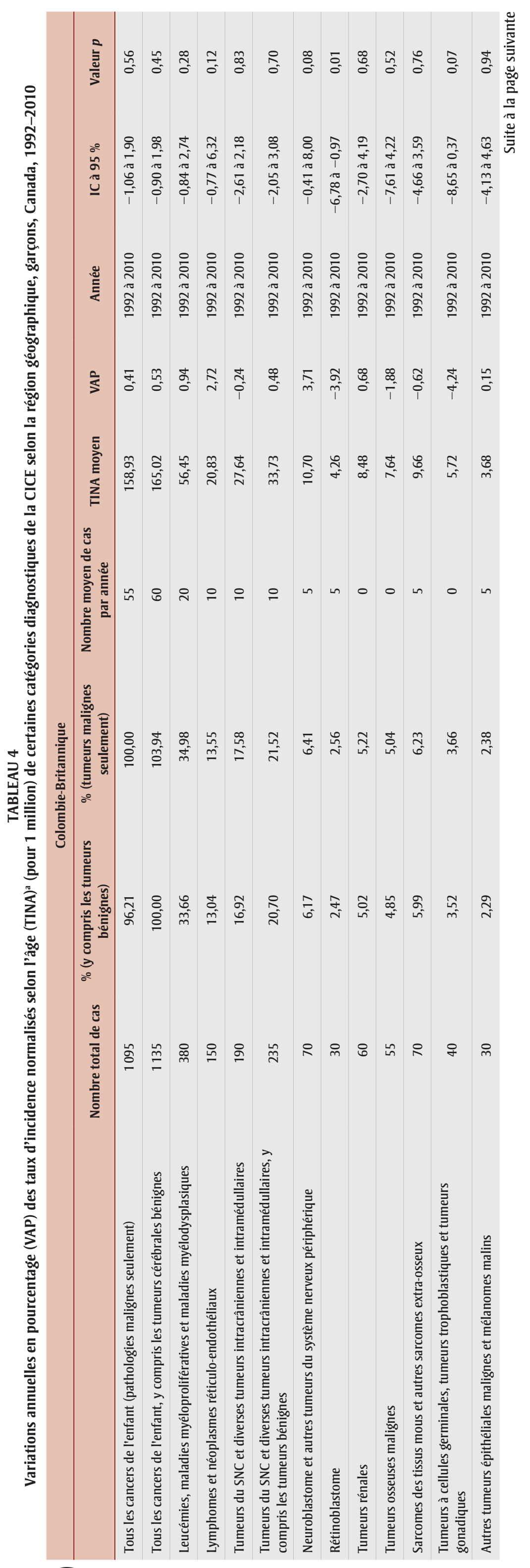




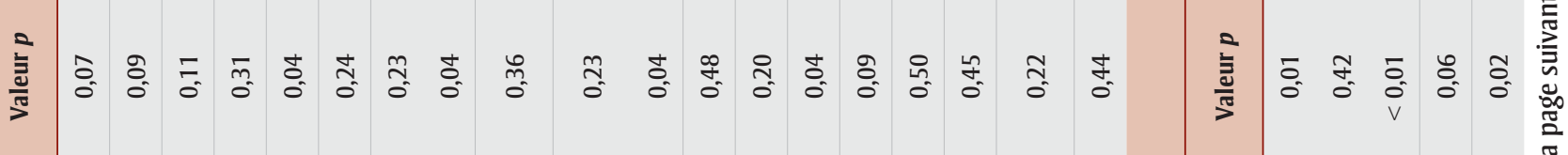

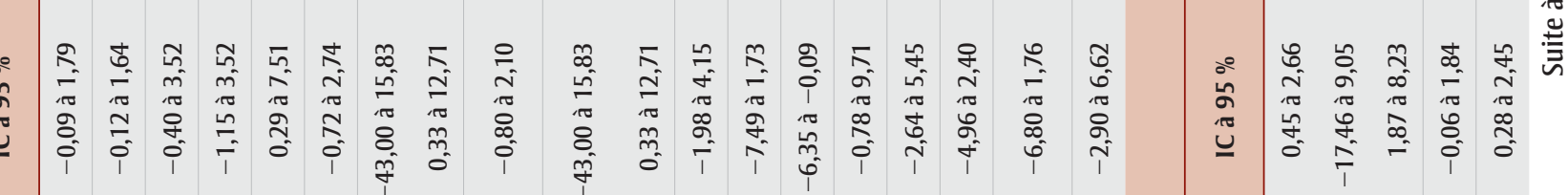

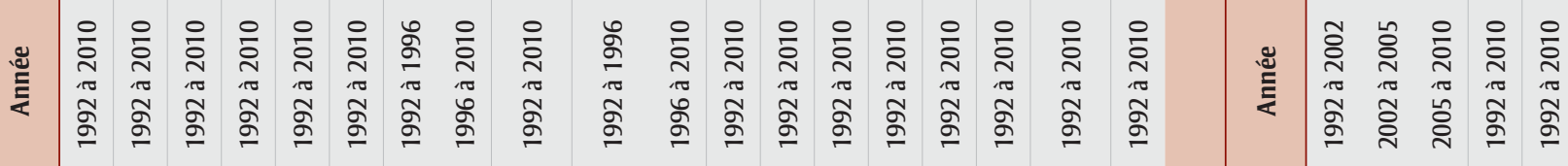

可

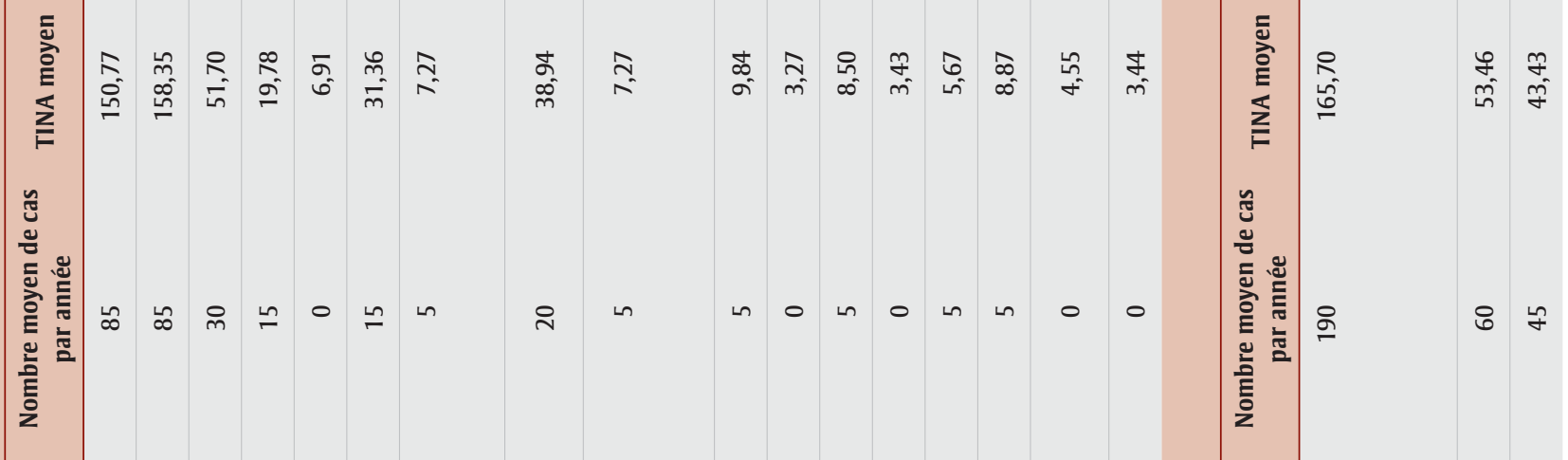

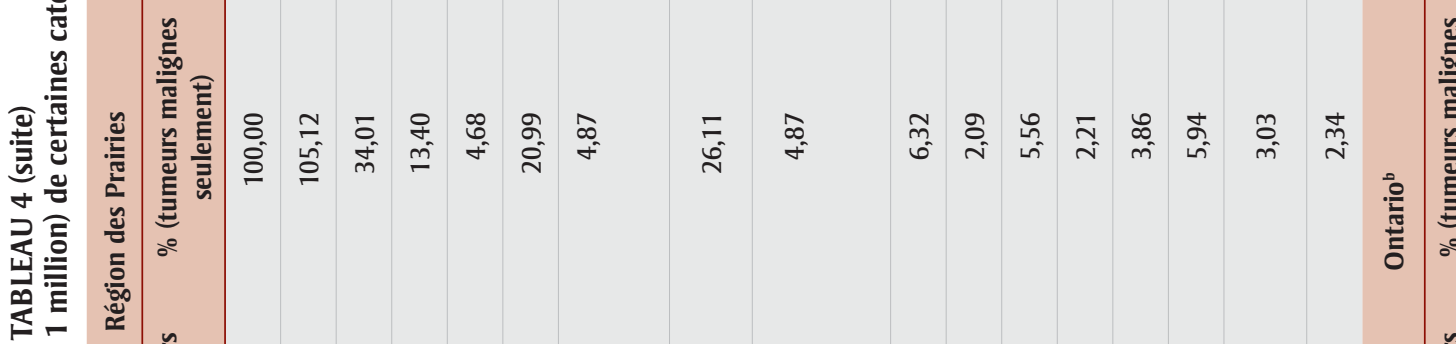

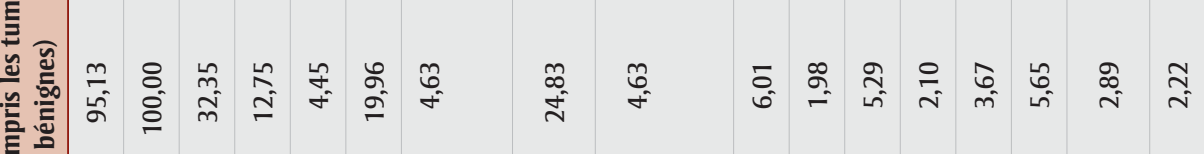

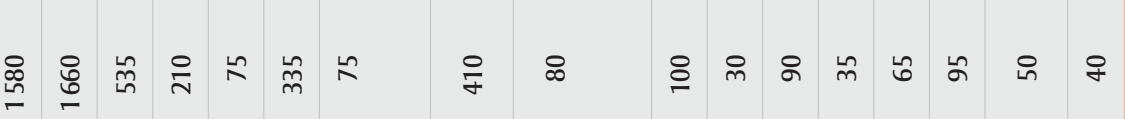

产

言

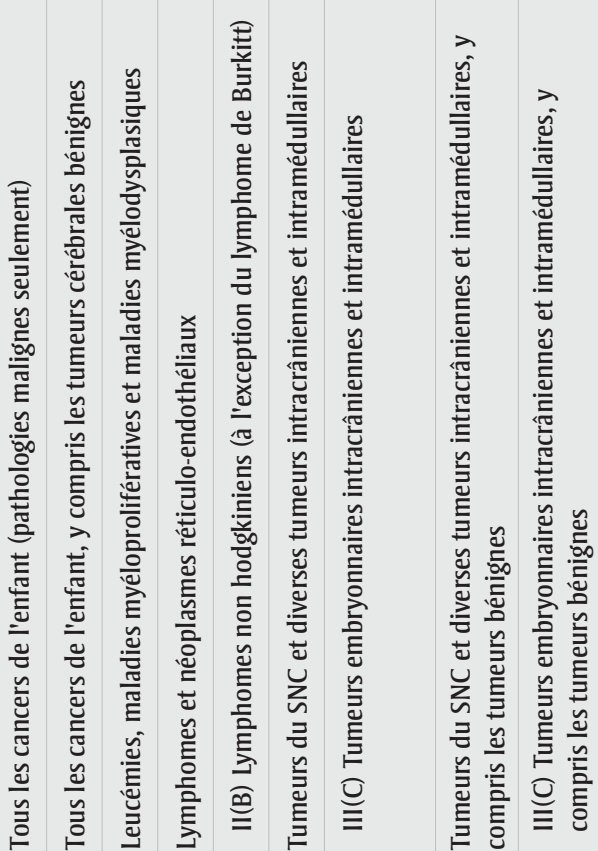

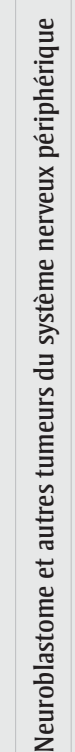

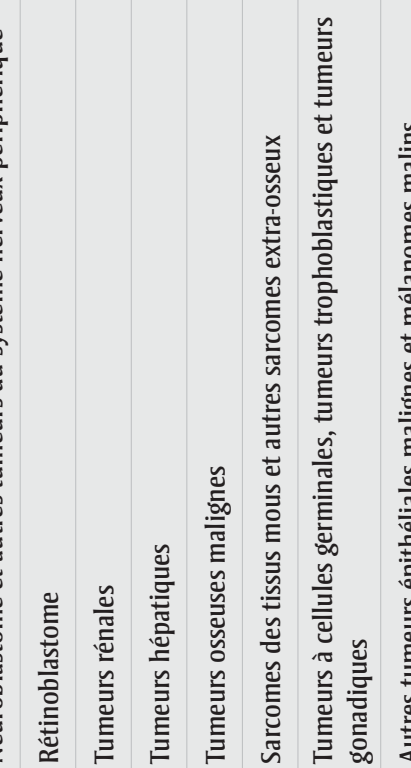

(1)

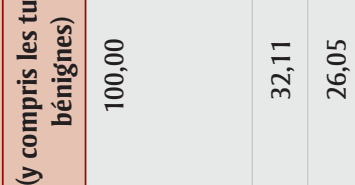

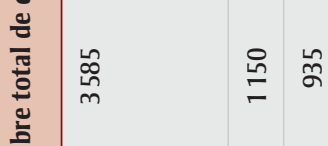

言

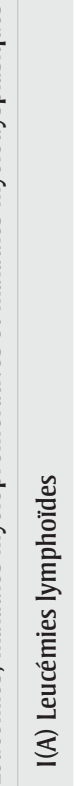




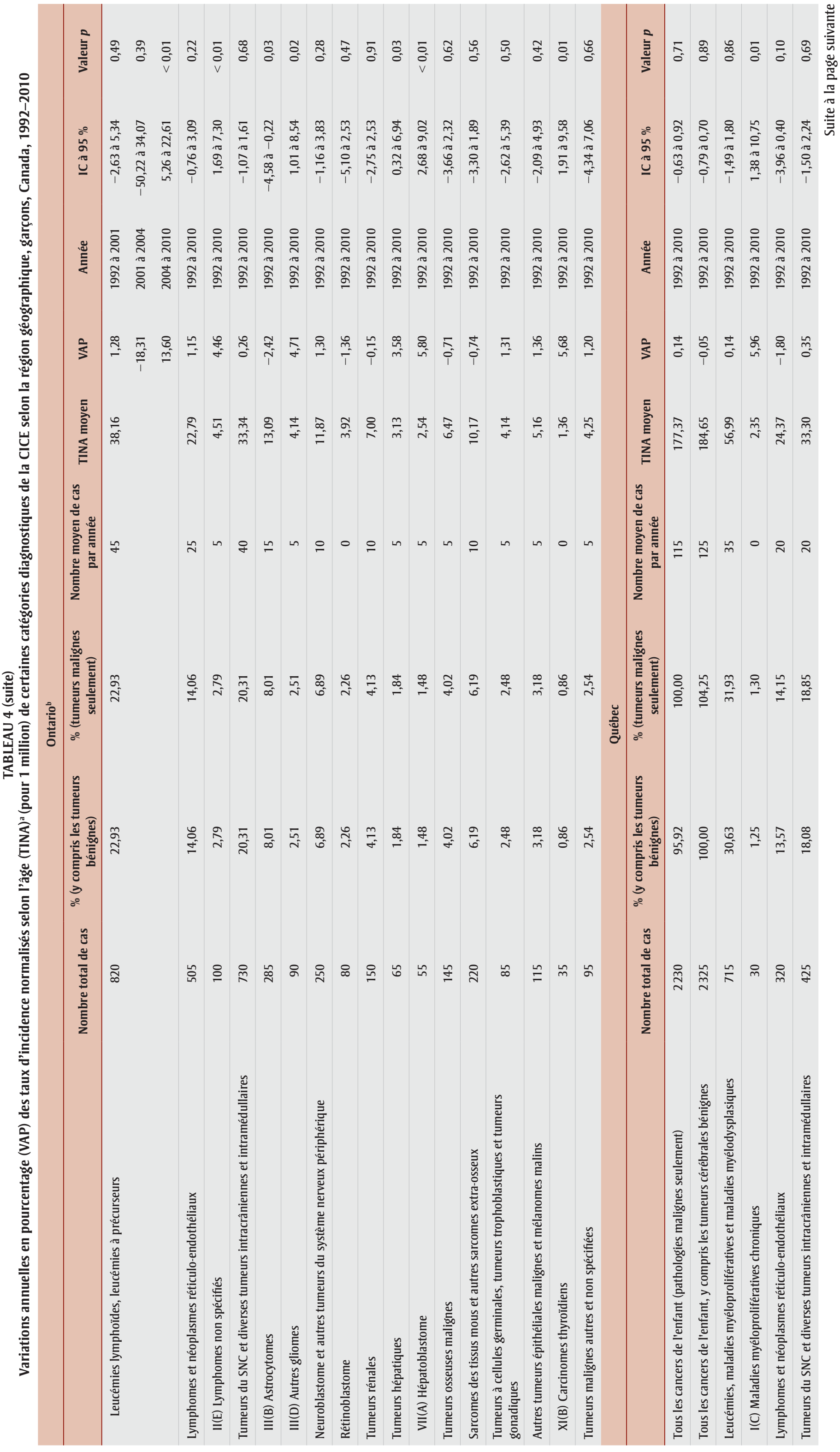




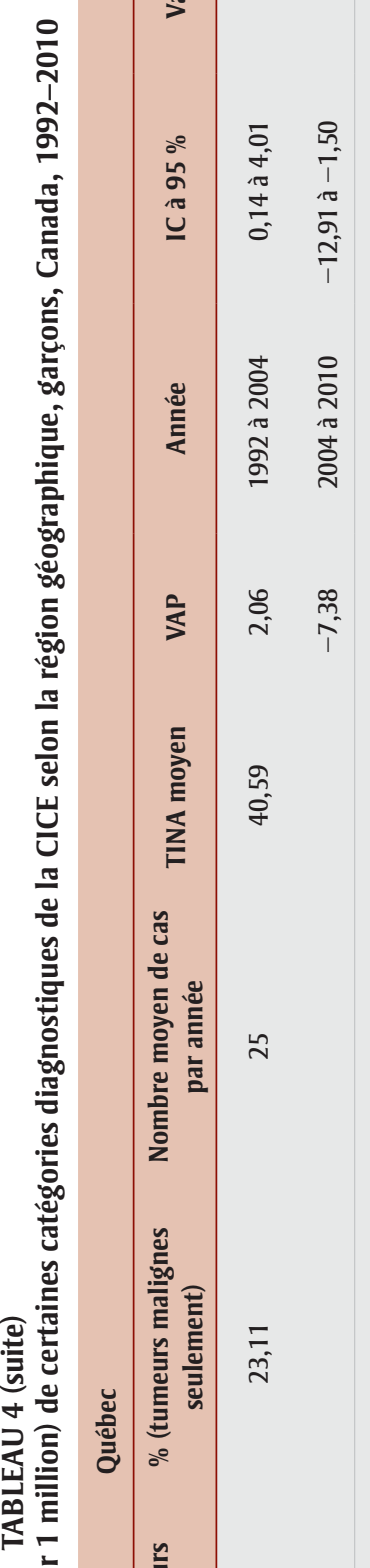

f

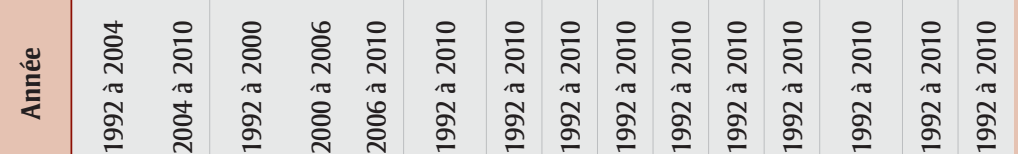

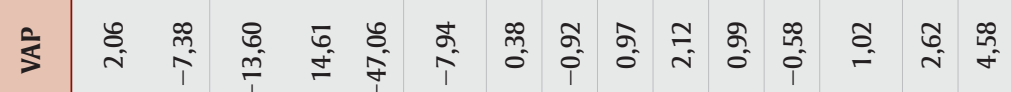

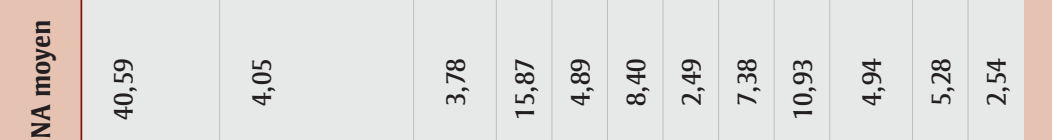

苋

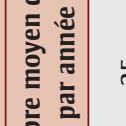

言

总

要

$=\stackrel{\sim}{\sim}$

产

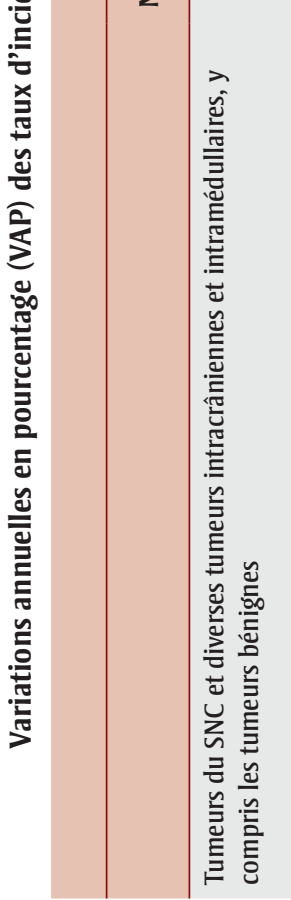

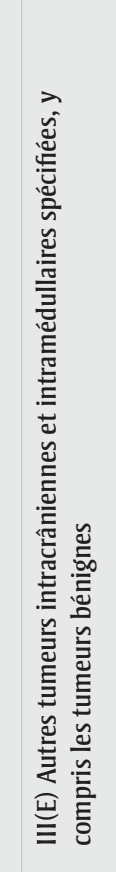

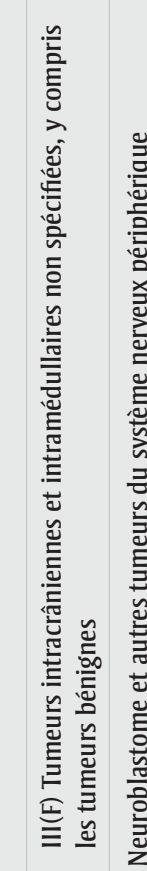

응
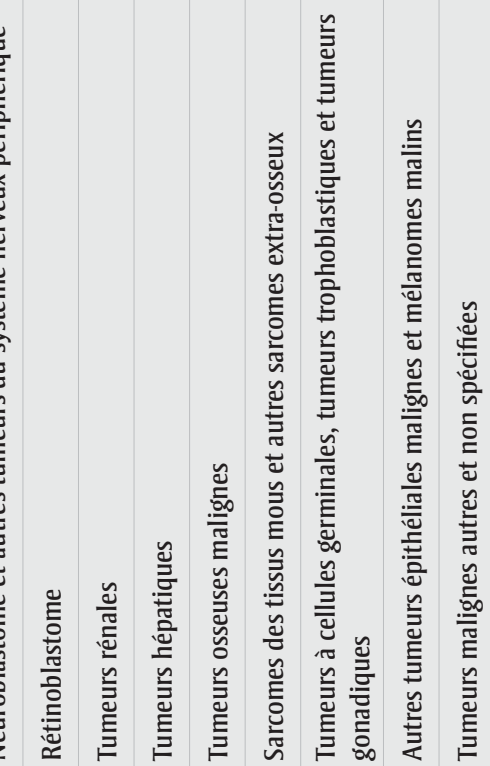

定

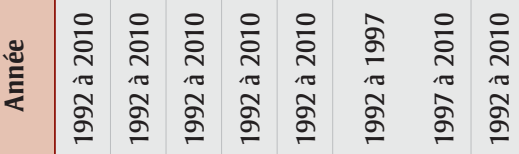

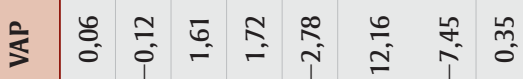

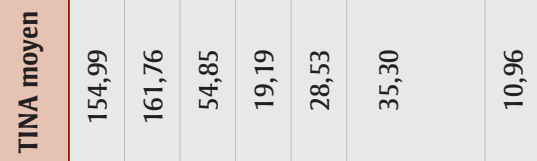

ฮั

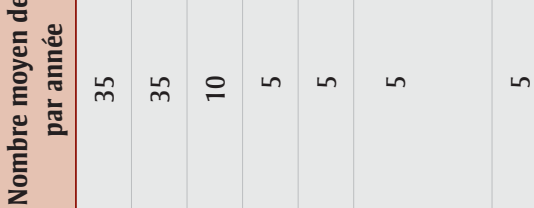

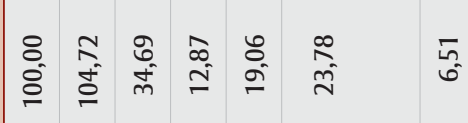

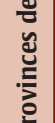

言

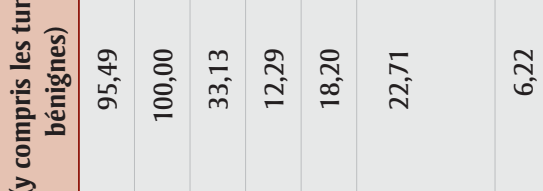



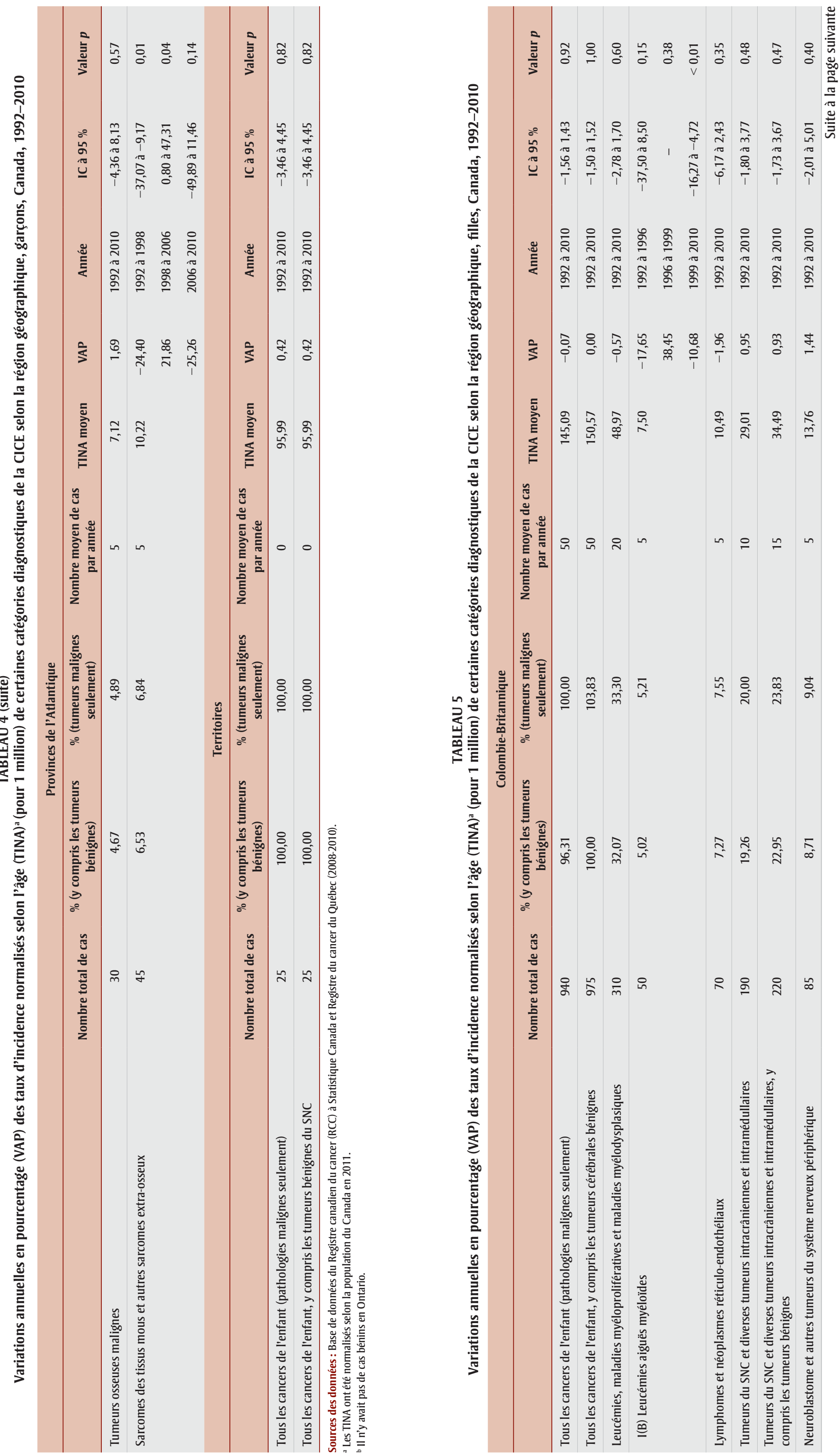


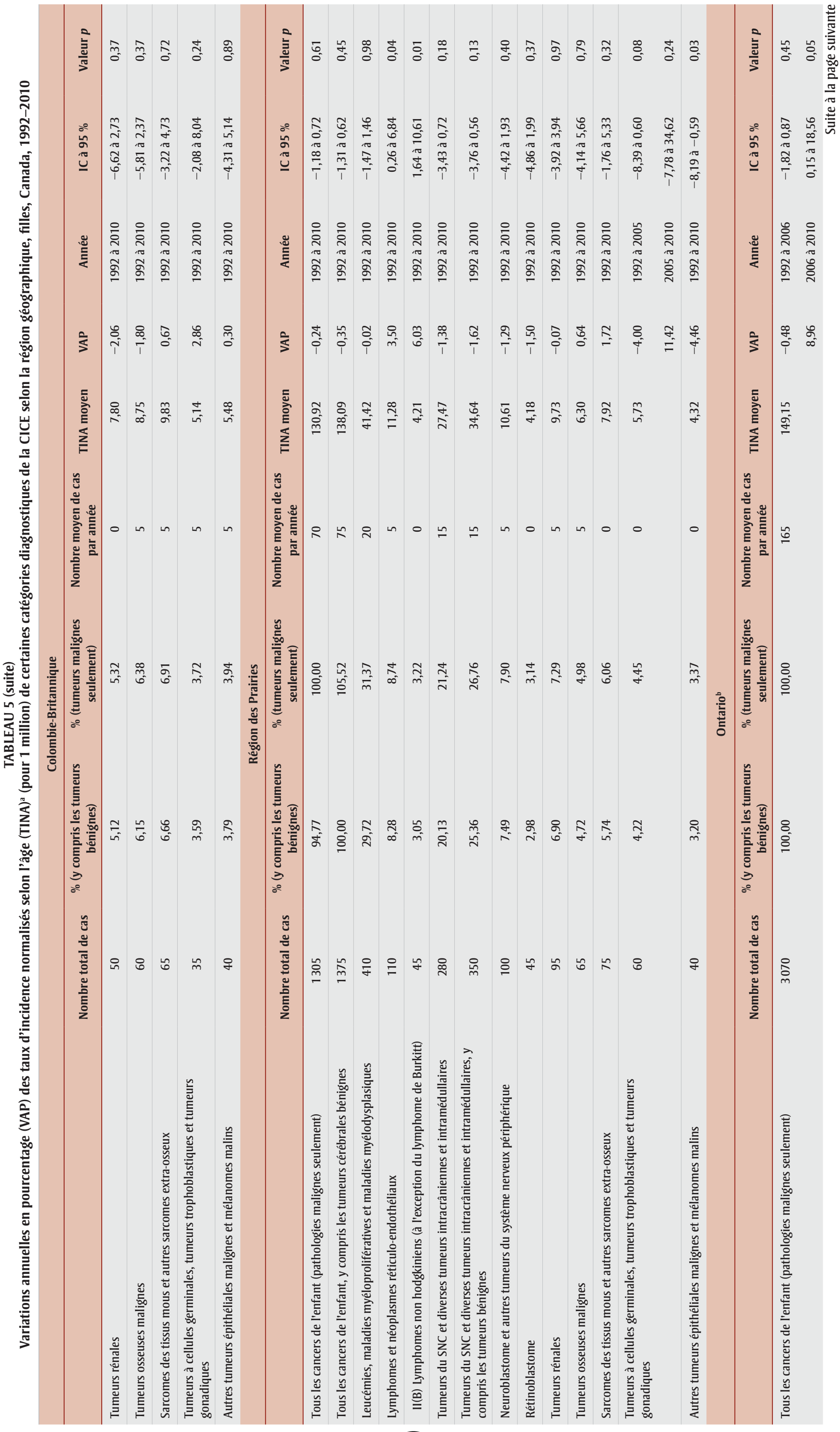




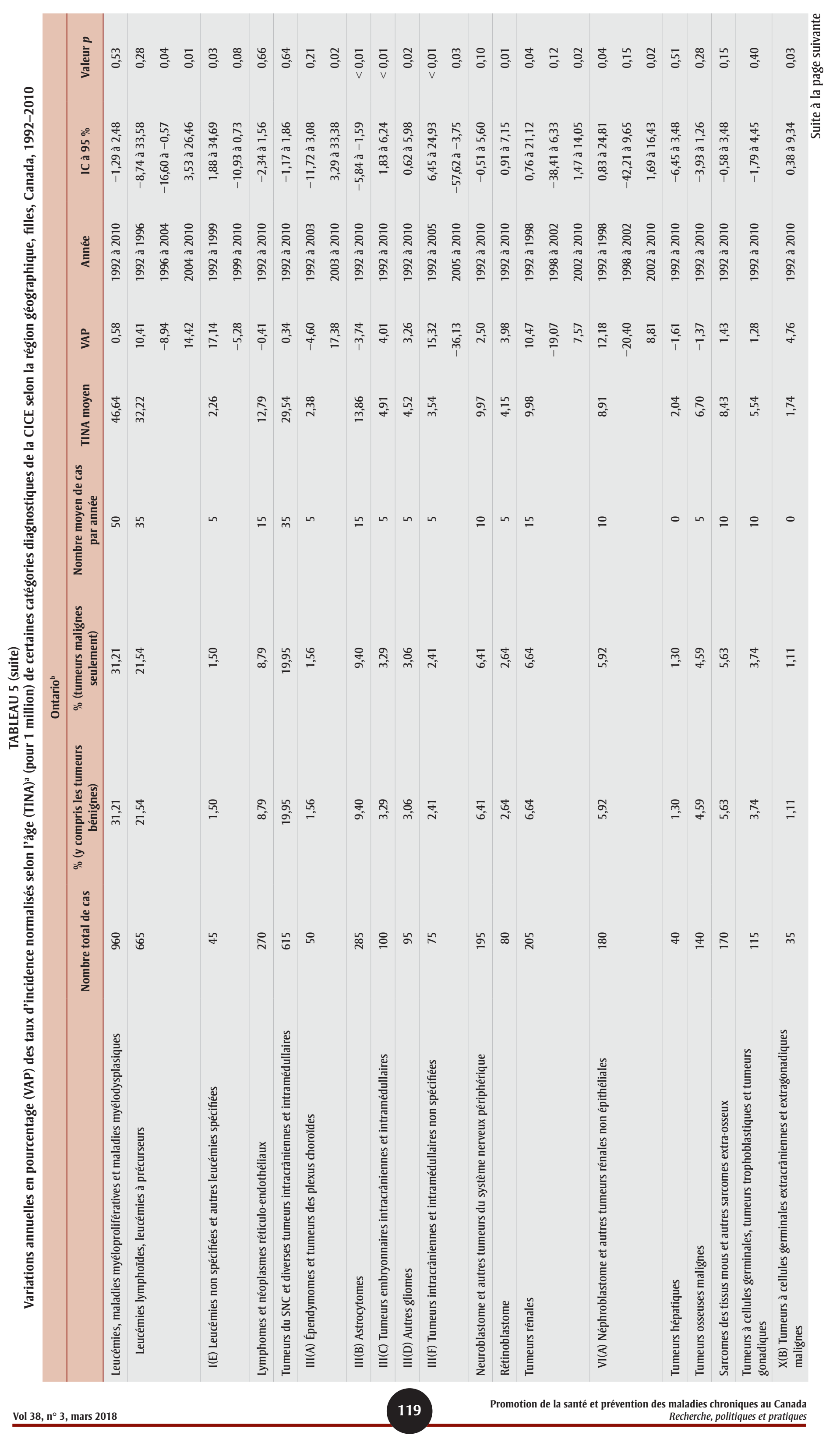




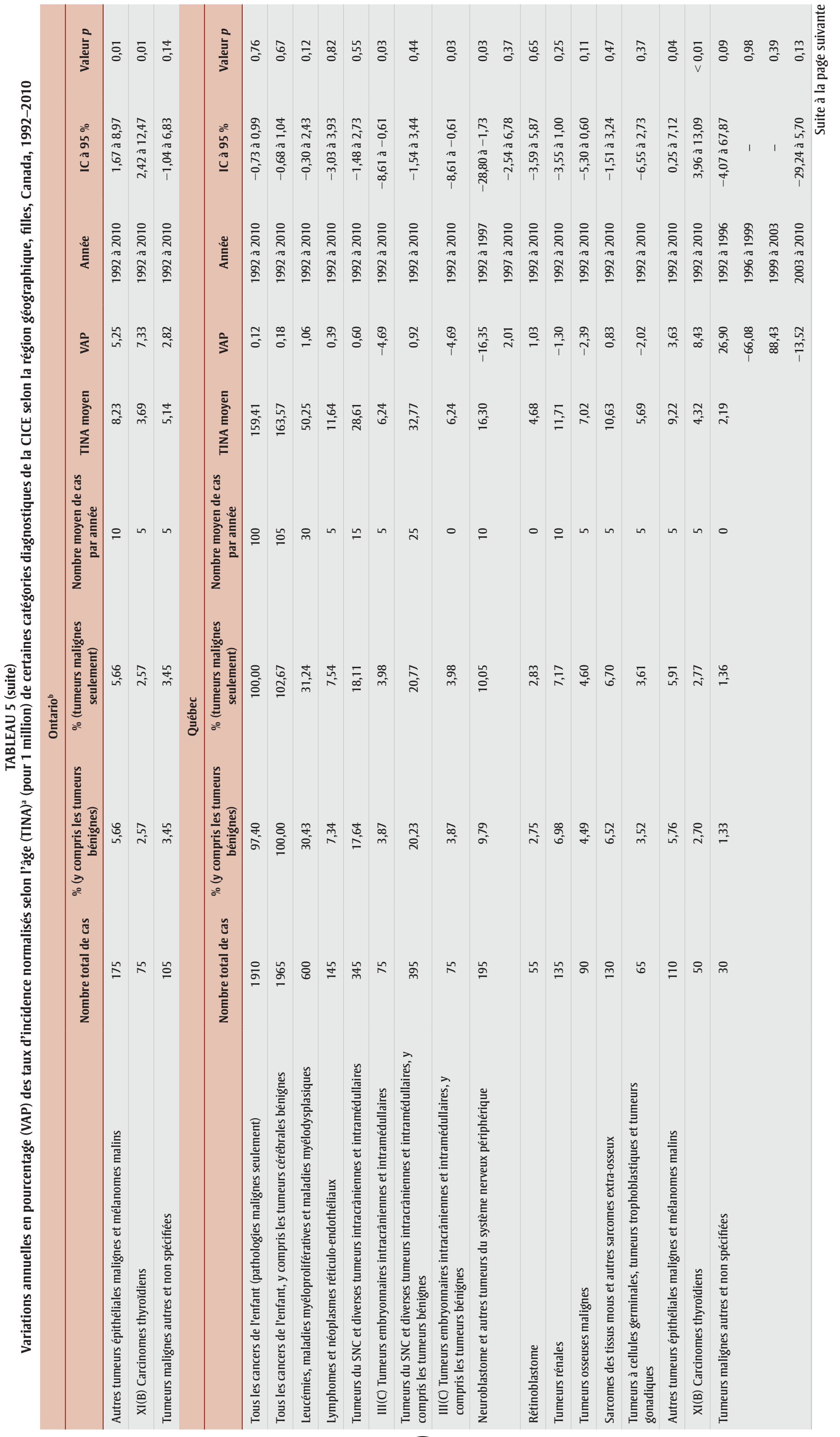




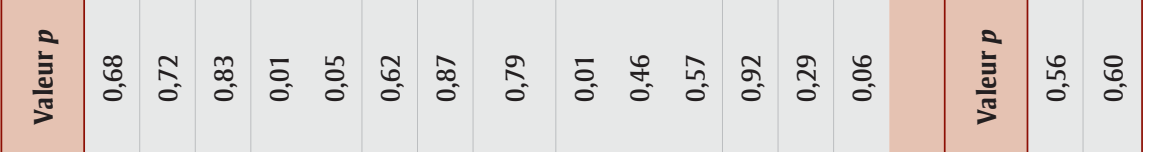

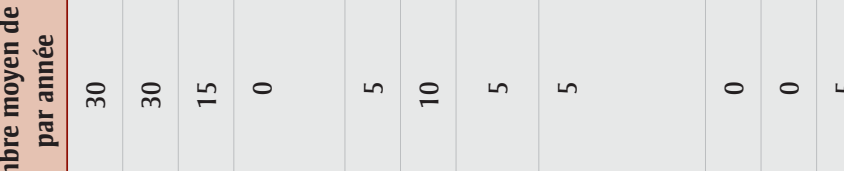

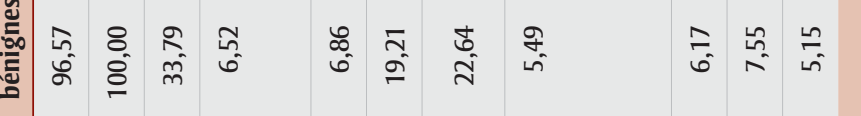

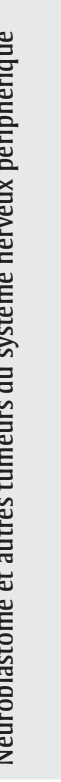

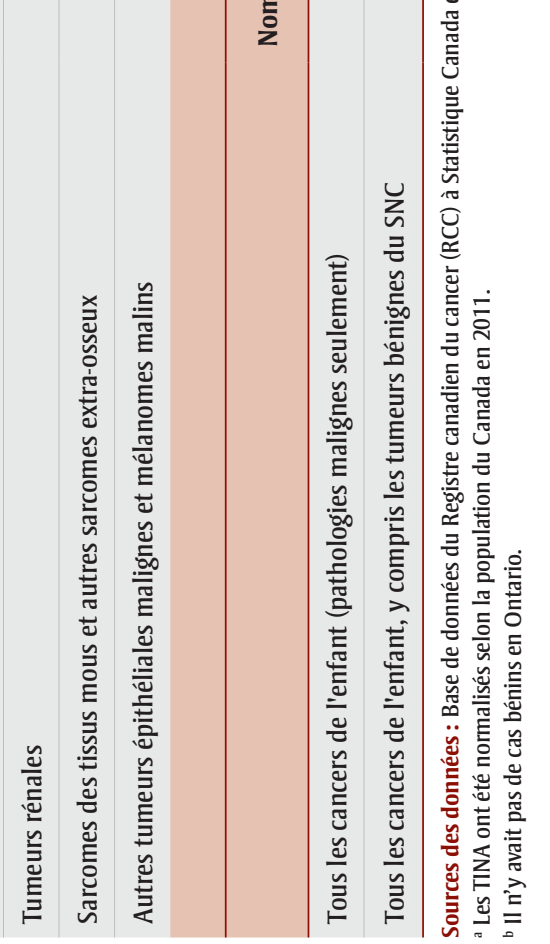




\section{FIGURE 2}

Taux d'incidence normalisés selon l'âge (TINA) annuels moyens (pour 1000 000) de tous les cancers confondus et des cancers les plus courants (\%) selon la région chez les enfants de moins de 15 ans, Canada, 2006-2010

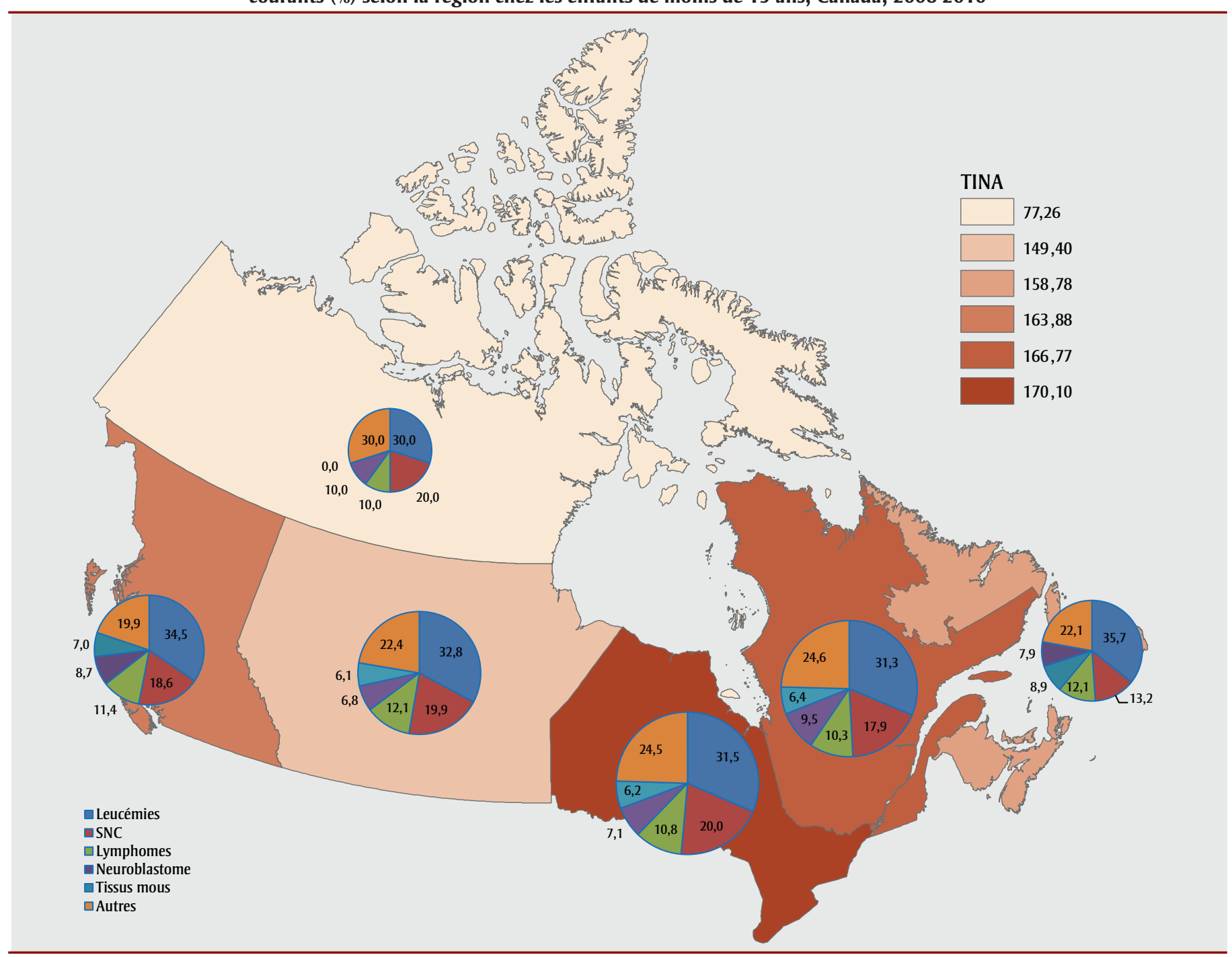

Sources des données : Base de données du Registre canadien du cancer (RCC) à Statistique Canada et Registre du cancer du Québec (2008 à 2010).

Remarques : 1 . Les diagrammes en secteurs représentent la répartition en pourcentage des nouveaux cas de cancer dans chaque région.

2. Les TINA ont été normalisés selon la population du Canada en 2011.

nombre de cas pour certains types ont pu entraîner des fluctuations aléatoires importantes des taux malgré une tendance statistiquement significative. Les taux d'incidence du cancer chez les enfants ont augmenté en moyenne de 0,4\% par année (IC à $95 \%=0,1$ à 0,8$)$, passant de 154,8 pour 1 million d'enfants en 1992 à 169,7 pour 1 million en 2010 (tableau 1). La leucémie tous types confondus et la leucémie lymphoïde plus spécifiquement ont connu une hausse tout aussi importante de 1992 à $2010($ VAP $=0,6 \%$, IC $=0,1$ à 1,2$)$. La leucémie lymphoïde est le type le plus répandu chez les enfants. Elle constitue près des quatre cinquièmes $(78,5 \%)$ de toutes les leucémies et, de ce fait, elle détermine largement le profil d'incidence de la leucémie tous types confondus. Les taux qui ont augmenté d'au moins $2 \%$ par année au cours de la période d'étude étaient les suivants : lymphomes non spécifiés (VAP $=3,4 \%, \mathrm{IC}=0,7$ à 6,2 ), épendymomes (VAP $=2,3 \%, \mathrm{IC}=0,2$ à 4,3 ), hépatoblastome (VAP $=2,4 \%, \mathrm{IC}=0,4$ à 4,4 ), carcinomes (VAP $=2,5 \%, \mathrm{IC}=0,2$ à 4,7 ), cancer de la thyroïde (VAP $=4,2 \%$; $\mathrm{IC}=1,4$ à 7,1 ) et mélanome (VAP $=2,7 \%$, $\mathrm{IC}=0,1$ à 5,4$)$. Les données laissent envisager une diminution des tumeurs à cellules germinales gonadiques malignes $(\mathrm{VAP}=-2,3 \%, \mathrm{IC}=-4,4$ à $-0,03) . \mathrm{La}$ figure 3 présente les tendances de tous les cancers confondus et des cinq cancers les plus courants chez les enfants de moins de 15 ans.

\section{Tendances selon le sexe}

Les tendances de tous les cancers confondus (VAP $=0,5 \%, \mathrm{IC}=0,2$ à 0,9 ) et des leucémies (VAP $=0,8 \%, \mathrm{IC}=0,03$ à 1,6 ) chez les garçons correspondaient aux augmentations observées dans l'ensemble (tableau 1). Une rupture de la tendance a été observée pour tous les cancers confondus chez les filles : le taux a augmenté de $3,2 \%$ par année (IC = 0,4 à 6,2) de 2004 à 2010 après une période initiale de stabilité. Des tendances positives ont également été observées pour d'autres hémopathies malignes sur l'ensemble de la période : divers néoplasmes lymphoréticulaires chez les garçons $(\mathrm{VAP}=6,8 \%, \mathrm{IC}=2,2$ à 11,7 ) 
FIGURE 3

Taux d'incidence normalisés selon l'âge (TINA) de tous les cancers confondus et des cinq cancers les plus courants chez les enfants de moins de 15 ans, Canada, 1992 à 2010

\section{TINA (pour 1000 000)}
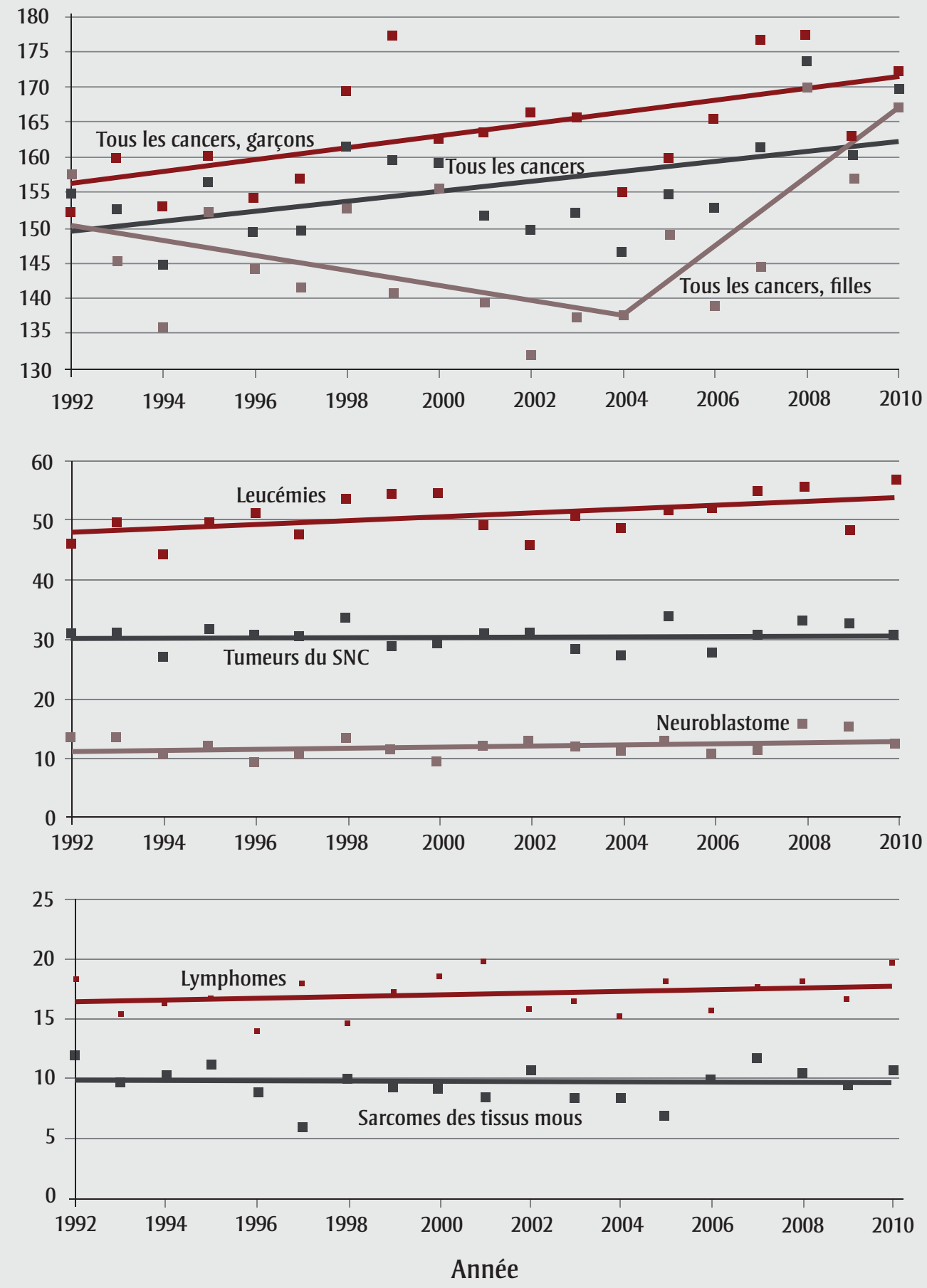

Sources des données : Base de données du Registre canadien du cancer (RCC) à Statistique Canada et Registre du cancer du Québec (2008-2010). Abréviations : SNC, système nerveux central; TINA, taux d'incidence normalisé selon l'âge.

Remarque : Les TINA ont été normalisés selon la population du Canada en 2011. 
et les filles (VAP $=4,6 \%$, IC $=0,7$ à 8,6) et lymphomes non spécifiés chez les garçons (VAP $=3,3 \%$; IC $=0,5$ à 6,2). Certaines tumeurs embryonnaires affichaient des tendances à la hausse chez les garçons. Il y a eu augmentation des neuroblastomes tous types confondus chez les garçons (VAP $=1,4 \%$, IC $=0,2$ à 2,6$)$, tout comme dans le sous-groupe des neuroblastomes et des ganglioneuroblastomes (IV(A)), qui constituaient presque tous les cas de neuroblastome chez les garçons. Les hépatoblastomes constituaient les quatre cinquièmes $(81,3 \%)$ de tous les cancers du foie chez les garçons, les taux d'hépatoblastome ayant augmenté de 3,2\% par année (IC $=0,6$ à 5,9) et étant à l'origine de la hausse de 2,2 \% par année des cancers du foie tous types confondus (IC $=0,01$ à 4,4$)$.

Même si les taux d'incidence des tumeurs du SNC sont demeurés stables, certaines de ses divisions ont connu des variations importantes. En particulier, les cas d'épendymome ont augmenté chez les filles $(\mathrm{VAP}=3,0 \%, \mathrm{IC}=0,6$ à 5,4), faisant écho à la transition des taux de cette maladie dans son ensemble. L'incidence des carcinomes a augmenté chez les filles $(\mathrm{VAP}=2,9 \%, \mathrm{IC}=0,6$ à 5,4$)$, tout comme son sous-groupe de cancer de la thyroïde $(\mathrm{VAP}=4,9 \%, \mathrm{IC}=1,8$ à 8,0). Dans le cas des tumeurs à cellules germinales gonadiques malignes, le taux a diminué chez les garçons (VAP $=-4,0 \%$, IC $=-6,7$ à $-1,2$ ) et une diminution non significative moins rapide a été observée chez les filles $(\mathrm{VAP}=-1,4 \%, \mathrm{IC}=-4,7$ à 2,1$)$.

\section{Tendances selon le groupe d'âge}

Une tendance globale à la hausse des cancers tous types confondus s'est dessinée chez les enfants de 1 à 4 ans (VAP $=0,9 \%$, IC $=0,4$ à 1,3 ) alors que les taux ont semblé stables dans les autres groupes d'âge (tableau 2). C'est plus précisément le taux d'incidence des leucémies lymphoïdes qui a augmenté chez les enfants de 1 à 4 ans $(\mathrm{VAP}=0,9 \%, \mathrm{IC}=0,1$ à 1,8$)$.

L'astrocytome était le plus grand sousgroupe des tumeurs du SNC tous types confondus, constituant plus des deux cinquièmes $(45,0 \%)$ du total. L'incidence de l'astrocytome augmentait avec l'âge, passant de 32,4\% chez les nourrissons à $52,4 \%$ chez les 10 à 14 ans. Les taux d'astrocytome ont diminué de 2,1\% par année chez les 10 à 14 ans (IC $=-3,7$ à
$-0,5)$ et sont demeurés stables chez les enfants de moins de 10 ans pendant toute la période à l'étude. En accord avec la tendance observée dans l'ensemble et chez les filles, les taux d'épendymomes ont augmenté chez les nourrissons (VAP $=5,6 \%$, $\mathrm{IC}=1,9$ à 9,4$)$ et chez les 10 à 14 ans (VAP $=5,1 \%$, IC $=1,5$ à 8,9, ) quoique les taux soient basés sur un petit nombre de cas.

Les tendances de l'incidence de plusieurs types de tumeurs embryonnaires variaient en fonction de l'âge. Les taux de neuroblastomes tous types confondus ont augmenté de 1,6\% par année chez les enfants de 1 à 4 ans (IC = 0,2 à 3,1), tous comme ceux du neuroblastome et du ganglioneuroblastome (IV(A)). L'hépatoblastome expliquait presque tous les cas de cancer du foie chez les moins de 5 ans. Chez les enfants de 1 à 4 ans, les taux d'hépatoblastome ont augmenté de 3,7\% par année (IC = 1,1 à $6,4)$.

\section{Tendances selon la région}

Les tendances par région sont présentées pour les deux sexes ensemble (tableau 3) et séparément (tableaux 4 et 5). C'est en Ontario que les taux combinés de tous les cancers confondus ont augmenté le plus depuis $2006(\mathrm{VAP}=5,9 \%, \mathrm{IC}=1,9$ à 10,1), après une période stable, et ils ont augmenté de façon non significative dans les autres régions entre 1992 et 2010. Des tendances positives ont été observées en Ontario pour les deux sexes : tandis que la tendance chez les filles était très semblable à celle observée dans l'ensemble, les tendances observées chez les garçons ont augmenté entre 1992 et 2002 (VAP $=1,6 \%$, $\mathrm{IC}=0,5$ à 2,7), et plus rapidement encore entre 2005 et 2010 (VAP $=5,0 \%$, IC $=1,9$ à 8,2).

Certaines cancers lymphohématopoïétiques affichaient des tendances à la hausse en Ontario et dans les Prairies : leucémies lymphoïdes chez les garçons (VAP $=1,4 \%$, $\mathrm{IC}=0,3$ à 2,5$)$ et chez tous les enfants $(\mathrm{VAP}=1,3 \%, \mathrm{IC}=0,2$ à 2,4$)$ et lymphomes non spécifiés (VAP $=4,3 \%, \mathrm{IC}=1,3$ à $7,5)$ en Ontario; lymphomes chez les filles $(\mathrm{VAP}=3,5 \%, \mathrm{IC}=0,3$ à 6,8$)$ et lymphomes non hodgkiniens (à l'exception du lymphome de Burkitt) chez les garçons et les filles ensemble $(\mathrm{VAP}=4,8 \%, \mathrm{IC}=1,6$ à 8,1 ) et séparément (garçons : VAP $=3,8 \%$, $\mathrm{IC}=0,3$ à 7,5 ; filles : VAP $=6,0 \%, \mathrm{IC}=1,6$ à 10,6) dans les Prairies. Deux points de jonction semblent indiquer un changement de direction de la tendance d'un sousgroupe de la leucémie lymphoïde, soit la leucémie lymphoblastique à précurseurs en Ontario chez les deux sexes, séparément et ensemble : une hausse non significative en début de période et une augmentation significative récente plus rapide depuis 2004.

Des tendances amphidirectionnelles de l'incidence des tumeurs du SNC ont été observées dans certaines régions. Les taux de tumeurs du SNC en Ontario ont diminué de façon non significative de 1,4\% par année de 1992 à 2004 (IC $=-2,8$ à 0,1 ), puis ils ont significativement augmenté de 5,0\% par année de 2004 à 2010 (IC = 1,0 à 9,2). Les taux dans la région de l'Atlantique affichaient quant à eux une tendance inverse : les TINA des tumeurs du SNC dans la région de l'Atlantique étaient les plus élevés au Canada entre 2002 et 2004, puis ils ont chuté au minimum en 2005 et entre 2007 et 2010 (données non présentées). L'incidence de l'astrocytome en Ontario a constamment diminué pendant la période à l'étude chez les garçons (VAP $=-2,4 \%$, IC $=-4,6$ à $-0,2$ ) et les filles (VAP $=-3,7 \%$, IC $=-5,8$ à $-1,6)$, mais il y a eu augmentation des épendymomes (VAP $=3,3 \%$, IC $=0,7$ à 6,1 ), des tumeurs embryonnaires intracrâniennes et intramédullaires chez les filles (VAP $=4,0 \%, \mathrm{IC}=1,8$ à 6,2 ) et d'autres gliomes chez les garçons et les filles dans leur ensemble (VAP $=4,5 \%$, $\mathrm{IC}=2,3$ à 6,7 ) et séparément (garçons : $\mathrm{VAP}=4,7 \%$, IC $=1,0$ à 8,5 ; filles : $\mathrm{VAP}=3,3 \%, \mathrm{IC}=0,6$ à 6,0 ).

Des changements significatifs ont également été observés concernant d'autres tumeurs embryonnaires dans le centre du Canada. Le taux de neuroblastomes chez les filles au Québec a diminué significativement de 16,4 \% par année de 1992 à 1997, mais il a augmenté de façon non significative de $2 \%$ par la suite. En ce qui concerne le neuroblastome chez les garçons au Québec, aucun point de jonction n'a été suggéré dans le modèle le mieux ajusté, mais un modèle à un point de jonction a révélé une tendance semblable, mais non significative, à celle observée chez les filles : les taux ont chuté de 7,0 \% (IC $=-22,6$ à 11,7) par année entre 1992 et 1997, puis ils ont augmenté de 2,6\% (IC $=-2,1$ à 7,5) (données non présentées). Le taux d'incidence du rétinoblastome a augmenté de $4 \%$ par année (IC = 0,9 à 
$7,2)$ pendant toute la période chez les filles en Ontario. Deux ruptures de la tendance montrent qu'il y a eu des hausses significatives de l'incidence des néphroblastomes chez les filles en Ontario au début de la période (dans les années 1990) et plus récemment (depuis 2002), et une tendance correspondante a été observée pour l'ensemble des tumeurs rénales. Toutefois, il semble que les tumeurs rénales chez les garçons ont diminué de 3,3\% par année (IC $=-6,4$ à $-0,1)$ dans les Prairies.

La hausse des carcinomes s'est révélée similaire en Ontario et au Québec, surtout en raison de l'augmentation des cancers de la thyroïde, plus particulièrement chez les filles. Au Québec, le cancer des os a diminué de 4,9\% (IC $=-8,5$ à $-1,2$ ) par année de 1992 à 2002 chez les garçons et les filles ensemble et il a augmenté de $6,2 \%$ (IC $=0,4$ à 12,3 ) par la suite.

\section{Analyse}

Notre étude a révélé que les taux d'incidence des cancers de l'enfant ont augmenté en moyenne de 0,4\% par année de 1992 à 2010. Des augmentations analogues ont été constatées aux États-Unis ${ }^{5}$, en Australie $^{6}$, dans les pays européens ${ }^{7}$, les nations asiatiques ${ }^{8}$ et à l'échelle internationale ${ }^{16}$. Une étude fondée sur les données du programme Surveillance, Epidemiology and End Results (SEER) du National Cancer Institute a révélé que les taux globaux d'incidence du cancer ont augmenté de façon non significative de 0,4\% par année entre 1992 et 2004 aux États-Unis ${ }^{5}$, ce qui correspond à l'ampleur de nos variations. Une augmentation non significative a également été observée (VAP $=0,3 \%$, IC $=-0,1$ à 0,7 ) entre 2001 et 2009 , d'après des données assurant une plus grande couverture de la population ${ }^{17}$. Si l'on compare les résultats obtenus par Ellison et ses collaborateurs pour tous les cancers confondus (ces auteurs ayant examiné les cinq cancers les plus courants à l'échelle nationale au cours de la même période ${ }^{4}$ ) et les nôtres, on observe des similarités pour les garçons et pour les deux sexes combinés, mais nous avons constaté une augmentation substantielle récente chez les filles.

Pour la période 2001-2010, notre étude a révélé une augmentation annuelle des taux globaux de $1,5 \%$ (IC = 0,6 à 2,4), surtout attribuable à l'augmentation des taux de cancer chez les filles (VAP $=2,5 \%$,
IC $=1,2$ à 3,8) (données non présentées). Cette tendance globale chez les filles est due en grande partie à la hausse des taux de leucémies (VAP $=2,3 \%, \mathrm{IC}=0,5$ à 4,2), de lymphomes (VAP $=1,8 \%$, IC $=-1,9$ à 5,6), de neuroblastome (VAP $=3,7 \%$, IC $=-0,8$ à 8,5$)$ et de sarcome des tissus mous (VAP $=3,9 \%, \mathrm{IC}=-0,8$ à 8,8 ), et surtout à une hausse marquée du taux de cancer de la thyroïde (VAP $=10,4 \%$, $\mathrm{IC}=3,4$ à 17,8) (données non présentées). Pour une période antérieure (1985-1992), Santé Canada a indiqué que les taux d'incidence de tous les cancers confondus chez les enfants et les adolescents de moins de 20 ans avaient tendance à augmenter légèrement ${ }^{18}$.

Les grandes similitudes de l'augmentation des TINA de certains cancers soulèvent des questions quant à la possibilité de causes communes, étant donné que les causes du cancer chez les enfants sont globalement inconnues. Plusieurs hypothèses ont été proposées pour expliquer ces tendances. Les changements peuvent s'expliquer en partie par des modifications apportées à la classification, à l'utilisation accrue de techniques spécialisées de diagnostic et à une meilleure déclaration des cas de cancer. Les augmentations globales ont été limitées aux périodes 1992-1999 et 2003-2010 et correspondaient aux tendances des leucémies, des lymphomes, du sarcome des tissus mous et des tumeurs du SNC (données non présentées). Les augmentations entre 1992 et 1999 coïncident avec la publication de la CICE en 1996 et l'utilisation accrue de l'imagerie par résonance magnétique (IRM) entre 1990 et 2001, et la hausse observée entre 2003 et 2010 coïncide avec la publication de la CIM-O-3 en 2001 et l'utilisation accrue de tests moléculaires en sus du diagnostic histopathologique visant à améliorer la précision et l'objectivité de ce dernier. Les tendances de l'incidence chez les enfants ont aussi été associées à des modifications de l'exposition à l'environnement, aux interactions entre gène et environnement, au mode de vie parental, au poids à la naissance et à des structures sociales ${ }^{7}$.

Les tendances à la hausse de l'incidence pourraient être en partie dues à une survie prolongée. Le pronostic s'est en effet amélioré au cours des 30 dernières années grâce à des diagnostics plus exacts et à de meilleures stratégies de traitement. En effet, des recherches ont montré que le risque de néoplasme malin subséquent est plus élevé chez les survivants d'un cancer de l'enfant que le risque de cancer chez les personnes du même âge dans la population générale ${ }^{19}$. Nos données montrent que le pourcentage de deuxièmes ou de troisièmes cancers est passé de 0,7 \% en 1992 à 4,1\% en 2006 (avec une interruption en 2004) puis a chuté considérablement chez les garçons, ainsi que chez les filles mais dans une moindre mesure (données non présentées). Ces hausses des néoplasmes malins subséquents au Canada coïncident avec l'ampleur et l'importance des hausses dans les tendances de l'incidence globale.

Un lien a été établi entre le risque de cancer chez l'enfant et l'âge de la mère à la naissance. Une vaste étude cas-témoins menée aux États-Unis a fait état d'une élévation de $8 \%$ du risque global de cancer chez les enfants pour chaque hausse de cinq ans de l'âge de la mère, les augmentations étant similaires pour la plupart des cancers fréquents ${ }^{20}$. L'âge de la mère pourrait également constituer l'indicateur d'expositions environnementales inconnues qui pourraient avoir changé au fil du temps ${ }^{6}$. $\mathrm{Au}$ Canada comme dans la plupart des pays développés, l'âge maternel moyen au premier et à l'ensemble des accouchements a augmenté depuis le milieu des années $1970^{21}$. Au cours de notre période à l'étude, l'âge moyen à l'ensemble des accouchements est passé de 27,9 ans en 1992 à 30,1 ans en $2010^{21}$. Cette augmentation de l'âge maternel a peut-être contribué à la hausse de l'incidence mais nous ignorons dans quelle mesure.

Le cancer chez l'enfant se caractérise par son hétérogénéité, les différents cancers ayant probablement des causes différentes. Pour faire suite à nos résultats, il serait utile de déterminer les types de tumeurs et les groupes de populations précisément touchés par ces tendances. L'augmentation la plus marquée des TINA de tous les cancers confondus s'observe chez les enfants de 1 à 4 ans. Cette hausse est attribuable en grande partie à l'augmentation de la leucémie, le cancer le plus courant chez les enfants (le tiers de tous les cancers). L'Ontario a connu entre 2006 et 2010 la hausse la plus marquée de tous les cancers confondus et de la leucémie, des sousgroupes de lymphomes, des tumeurs du SNC, des tumeurs embryonnaires, des carcinomes et du cancer de la thyroïde. Bien qu'il puisse exister des différences démographiques ou étiologiques entre régions, la variation dans les pratiques des registres du cancer pourrait également expliquer ces 
différences géographiques dans l'incidence du cancer.

Les leucémies tous types confondus et la leucémie lymphoïde en particulier ont connu une hausse significative équivalente. Le taux d'incidence de la leucémie lymphoïde a aussi augmenté significativement chez les enfants de 1 à 4 ans. Des augmentations similaires des leucémies ont été signalées dans d'autres pays développés ${ }^{5,6,22}$. Des études antérieures ont démontré que le rayonnement ionisant, certaines anomalies génétiques, un poids élevé à la naissance, les agents alkylants cytotoxiques, l'âge des parents, leur tabagisme, l'exposition aux pesticides avant et après la naissance, la pollution atmosphérique liée à la circulation résidentielle et l'exposition prénatale à des agents infectieux comme le virus John Cunningham ont été associés à la leucémie chez les enfants ${ }^{23-27}$. Les fœetus et les jeunes enfants peuvent être plus vulnérables aux expositions en raison du sous-développement de leurs mécanismes de détoxication ou des taux d'ingestion plus élevés par rapport à leur poids comparativement aux enfants plus âgés. Il existe un grand nombre de données probantes établissant une association positive entre l'amélioration du statut socioéconomique et un pic d'incidence de la leucémie aiguë lymphoblastique (LAL) à précurseurs $\mathrm{B}$ chez les enfants de 2 à 3 ans $^{28}$. Il a également été avancé qu'une réponse immunitaire aberrante à l'infection retardée par des agents inconnus pourrait jouer un rôle dans la conversion des clones préleucémiques en LAL à précurseurs B patente $^{23}$. La leucémie lymphoblastique à précurseurs a augmenté de façon non significative de 0,4\% par année (IC $=-0,6$ à 1,5$)$ chez les enfants canadiens de 1 à 4 ans de 1992 à 2010 (tableau 2) mais, en Ontario, on a observé une hausse significative de la maladie entre 2004 et 2010 seulement (tableau 3). Une étude spatiale canadienne a révélé que les régions où la proportion d'immigrants était plus forte présentaient des taux d'incidence plus élevés de leucémies chez les enfants ${ }^{29}$. La proportion d'immigrants au Canada est en hausse constante, étant passée de 16,1\% de la population en 1991 à 18,4 \% en 2001 et à $20,6 \%$ en $2011^{30}$. Le pourcentage d'immigrants établis en Ontario dépassait $50 \%$ de 1992 à $2006^{31}$, et ils constituaient $25,6 \%$ de la population de la province en $1996,26,8 \%$ en 2001 et $28,3 \%$ en $2006^{32}$. L'augmentation de la population d'immigrants pourrait jouer un rôle dans les hausses observées de l'incidence du cancer, mais cette association n'a été établie que dans une étude.

Le taux stable de tumeurs du SNC a aussi été observé aux États-Unis au cours de périodes de déclaration semblables (1992 à $2004^{5}$ et 1987 à $2009^{33}$ ). L'augmentation des tumeurs du SNC aux États-Unis, limitée à la période 2000-2010, est comparable à la tendance en Ontario ${ }^{22}$. De plus, une variation significative du taux de tumeurs cérébrales bénignes a été constatée dans la population américaine. Selon certains, l'augmentation serait probablement attribuable à la modification des méthodes de détection et de déclaration de ces mala$\operatorname{dies}^{34}$. L'augmentation récente des tumeurs du SNC en Ontario pourrait s'expliquer par l'utilisation accrue de marqueurs moléculaires pour compléter le diagnostic histopathologique.

Le Centre International de Recherche sur le Cancer (CIRC) a déclaré que les rayons $\mathrm{X}$ et les rayons gamma, deux types de rayonnement ionisant, sont les seuls facteurs de risque établis des cancers du $\mathrm{SNC}^{35}$. Le CIRC inclut également le rayonnement non ionisant des radiofréquences des télécommunications comme cause possible de tumeurs malignes du SNC, mais les données probantes sont limitées ${ }^{35,36}$. Les maladies génétiques et héréditaires sont associées à un risque accru. Les modifications de l'exposition environnementale et médicale et des interactions entre gène et environnement, comme le rayonnement ionisant et les pesticides, ont été associés aux récentes augmentations de l'incidence des tumeurs du $\mathrm{SNC}^{37}$. Une étude canadienne a constaté une association positive entre l'astrocytome et l'exposition maternelle à la pollution de l'air résidentiel ${ }^{24}$.

Notre étude montre que l'incidence de l'hépatoblastome a augmenté de 2,4\% par année entre 1992 et 2010. Une augmentation annuelle de $4 \%$ a été observée aux États-Unis entre 1992 et $2004^{5}$. Même si on a pu déterminer peu de causes à l'hépatoblastome, on dispose de plusieurs indices. Des études ${ }^{38-40}$ ont révélé une forte association entre l'hépatoblastome et un très petit poids à la naissance (TPPN) (moins de 1500 g), ce qui laisse entrevoir une étiologie iatrogène. Le risque d'hépatoblastome était 20 fois plus élevé chez les enfants ayant un TPPN et doublait chez les enfants ayant un poids modérément petit à la naissance (1 500 à $2500 \mathrm{~g})^{38}$. Des chercheurs avaient déjà observé que l'augmentation des cas d'hépatoblastome correspondait à une hausse de la fréquence du petit ou du très petit poids à la naissance aux ÉtatsUnis $^{41}$. Selon l'Agence de la santé publique du Canada, le taux de petit poids à la naissance a en général augmenté entre 2001 et 2010 au Canada ${ }^{42}$. En outre, le taux de survie des bébés de petit poids à la naissance au Canada s'est accru grâce à de meilleurs soins néonataux. Ensemble, ces facteurs pourraient expliquer la tendance à la hausse de l'hépatoblastome que nous avons observée.

Comme le confirment nos données, le neuroblastome est le cancer de l'enfant le plus couramment diagnostiqué chez les nourrissons $^{43}$, correspondant à $26,4 \%$ de tous les diagnostics de cancer au Canada. Il s'agit du troisième cancer en importance chez les enfants de 1 à 4 ans, rassemblant 10,5\% de tous les cas (figure 1). L'incidence du neuroblastome a augmenté significativement chez les enfants de 1 à 4 ans entre 1992 et 2010, tout comme en Europe ${ }^{43}$. L'utilisation accrue de techniques spécialisées de diagnostic permettant de détecter des tumeurs latentes ou asymptomatiques peut avoir contribué à cette augmentation de l'incidence ${ }^{44}$. Ainsi, la forte baisse des cas de neuroblastome observée au Québec entre 1992 et 1997 correspond à la fin d'un vaste essai de dépistage en 1994 qui a donné lieu à la détection de nombreux cas de neuroblastomes qui, autrement, n'auraient jamais été cliniquement détectés ${ }^{45}$.

D'autres études ont confirmé l'augmentation rapide du cancer de la thyroïde chez les enfants ${ }^{17,46}$. Selon Siegel et ses collaborateurs, les taux d'incidence du cancer de la thyroïde ont augmenté de 4,9\% par année (IC $=3,2$ à 6,6) chez les enfants et les adolescents (moins de 20 ans) des États-Unis entre 2001 et $2009^{17}$. Des études antérieures ont également révélé une hausse des taux de cancer de la thyroïde chez les adultes au Canada et dans d'autres pays $^{1,47,48}$. On ignore si les causes de l'augmentation des carcinomes de la thyroïde chez les enfants sont les mêmes que chez les adultes. L'utilisation accrue de techniques spécialisées de diagnostic a contribué à la détection de petites tumeurs infracliniques de la thyrö̈de ${ }^{49}$. L'utilisation plus fréquente de l'imagerie pour diagnostiquer les maladies thyrö̈diennes bénignes, plus courantes chez la femme que chez l'homme, pourrait expliquer la plus grande hausse des cas de cancer de la thyroïde chez la femme ${ }^{49}$. De plus, il a été démontré que l'exposition au rayonnement résultant 
d'une utilisation accrue de la tomodensitométrie $^{50}$ pouvait augmenter le risque de cancer de la thyroïde ${ }^{51,52}$. Des données probantes ont aussi révélé une association positive entre l'obésité et le risque de cancer de la thyroïde chez les adultes ${ }^{53,54}$. La prévalence accrue de l'obésité chez les enfants $^{55-57}$ pourrait expliquer en partie la hausse du cancer de la thyroïde.

La diminution annuelle significative de 2,1 \% de l'incidence de l'astrocytome chez les enfants de 10 à 14 ans est similaire à la diminution non significative (VAP $=-1,9$, IC $=-4,4$ à 0,8 ) observée aux États-Unis dans le même groupe d'âge entre 1992 et $2004^{5}$. La diminution des cas d'astrocytome pourrait s'expliquer en partie par l'amélioration du diagnostic et de la classification depuis l'adoption de la CIM-O-3 en 2001. Dans la CIM-O-3, l'astrocytome pilocytique est codifié comme une tumeur indéterminée ou à la limite de la malignité (code morphologique 9421/1), ce qui explique son exclusion de l'analyse des cas malins. En outre, la diminution de l'incidence des astrocytomes sans autre indication (SAI) laisse envisager une classification diagnostique plus précise des tumeurs du $\mathrm{SNC}^{33}$. La tendance à la baisse de l'incidence des tumeurs à cellules germinales gonadiques malignes concorde avec la diminution de la prévalence des anomalies congénitales ${ }^{20,58}$.

\section{Points forts et limites}

Nos résultats doivent être interprétés en tenant compte des limites et des points forts de notre étude. Bien que les registres du cancer des provinces et des territoires s'efforcent de répertorier et de définir les nouveaux cas de cancer en suivant la norme nationale, les procédures de déclaration et l'exhaustivité des données ne sont pas uniformes dans l'ensemble des registres $^{1}$. L'incidence de certains cancers au Québec, en particulier ceux dont le diagnostic repose avant tout sur l'examen anatomopathologique, est sous-estimée du fait de la dépendance du registre aux données d'hospitalisation pendant la période à l'étude. Même si tous les registres du cancer des provinces et des territoires enregistrent maintenant les cancers selon les règles du programme SEER pour de multiples cancers primitifs, ils n'ont pas tous été en mesure de le faire en respectant les nouvelles exigences de $2007^{9}$.
L'incidence du cancer pourrait être sousdéclarée dans certaines provinces en raison de l'absence d'information sur les cas pour lesquels on disposait d'un " certificat de décès seulement " (CDS) ou d'un jumelage incomplet des données sur le cancer avec l'information des statistiques de l'état civil pour les données utilisées dans notre étude. Ainsi, le nombre de cas de CDS de 2008 à 2010 à Terre-Neuve-etLabrador a été estimé d'après les données de 2007. Cette province a récemment mis en œuvre des processus de confirmation des décès afin de confirmer les cas et a amélioré la déclaration des cas dans les régions où on observait auparavant un sous-enregistrement. Au Québec, les cas de CDS ont été enregistrés de façon incomplète avant 2000. Le nombre de cas de CDS inscrit pour 2010 au Québec est la moyenne des données de 2005 à 2009. L'Ontario n'ayant pas déclaré de cas de CDS entre 2008 et 2010, le nombre de cas pour ces trois années dans la province a été estimé en calculant la moyenne des cas de CDS entre 2003 et 2007. Le nombre de cas de CDS est inférieur à $2 \%$ du nombre total de nouveaux cas.

Les tumeurs cérébrales bénignes ne sont pas systématiquement saisies ou déclarées au RCC, ce qui conduit à une sousdéclaration de ces cas dans le RCC d'après notre analyse (données non présentées). L'inclusion des tumeurs cérébrales bénignes dans l'analyse pourrait produire de fausses tendances si l'on comparait les incidences dans le temps et selon les régions, étant donné cette collecte incomplète des données. Par exemple, l'analyse fondée sur l'ensemble de données regroupant les tumeurs bénignes et les tumeurs malignes du SNC n'a pas permis de déceler la rupture statistiquement significative de la tendance du TINA de tous les cancers confondus chez les filles. Autre exemple : l'ajout des cas prépondérants de tumeurs bénignes (86 \%) au total des autres tumeurs intracrâniennes et intramédullaires spécifiées (III(E)) a donné lieu à une forte tendance de points de jonction chez les enfants de 5 à 9 ans (tableau 2).

Une erreur de type I peut avoir faussé les résultats des groupes diagnostiques comptant seulement un petit nombre de cas. Plusieurs tests ont été effectués avec ajustement pour neutraliser la probabilité générale d'erreur de surajustement de 0,05 mais du fait de ces petits effectifs, les fluctuations aléatoires des taux peuvent conduire à de fausses tendances significatives. Les tendances touchant un petit nombre de cas et celles dont les intervalles de confiance sont larges doivent donc être interprétées de façon critique. Par exemple, l'augmentation des lymphomes non hodgkiniens (à l'exception du lymphome de Burkitt) chez les filles dans les Prairies repose sur un petit nombre de cas (45) entre 1992 et 2010. Pour certains résultats significatifs, la signification est proche du seuil de 0,05, par exemple pour la diminution des tumeurs à cellules germinales gonadiques malignes et l'augmentation des cancers du foie chez les garçons. Ces tendances demandent donc à être validées.

La hausse de tous les cancers confondus et de certains néoplasmes variaient d'une région à l'autre en ampleur comme en signification. La signification statistique obtenue en Ontario pourrait s'expliquer par la taille de sa population.

Nous avons décrit dans notre étude les différences entre les tendances en fonction du type de tumeur, du sexe, de l'âge et de la région, mais nous n'avons pas analysé statistiquement les relations entre ces tendances, ce qui peut conduire à inclure des associations fallacieuses dans les résultats.

La principale force du RCC est la couverture complète de la population et l'excellente qualité des données. Notre analyse présente les tendances actuelles de l'incidence du cancer chez les enfants et, à notre connaissance, elle constitue le premier rapport concernant les groupes diagnostiques détaillés dans un contexte à la fois démographique et géographique.

\section{Conclusion}

En résumé, les taux globaux d'incidence du cancer chez les enfants ont lentement augmenté depuis 1992. Des hausses statistiquement significatives de plusieurs cancers tels la leucémie, les lymphomes non spécifiés, l'épendymome, l'hépatoblastome, le cancer de la thyroïde et le mélanome ont été observées. Les différences dans les tendances temporelles ont aussi été enregistrées selon le sexe, l'âge et la région. L'augmentation la plus importante des taux de tous les cancers confondus a eu lieu en Ontario et les taux ont augmenté de façon non significative dans les autres régions de 1992 à 2010. Autre nouvelle constatation : 
le taux d'incidence de l'astrocytome a significativement diminué chez les enfants de 10 à 14 ans. Compte tenu de notre compréhension limitée de l'étiologie des cancers chez l'enfant, notre étude révèle l'importance de la surveillance, qui fournit l'occasion de mieux comprendre les facteurs à l'origine des tendances de l'incidence. Ces connaissances pourraient un jour être utiles aux politiques et aux programmes en santé publique.

\section{Remerciements}

Nous sommes reconnaissants envers les registres provinciaux et territoriaux du cancer et la Division de la statistique de la santé de Statistique Canada, dont la collaboration nous a permis d'obtenir les données du Registre canadien du cancer (RCC). Nous remercions le Registre du cancer du Québec d'avoir fourni les données agrégées pour 2008-2010. Nos remerciements vont également à M. Robert Semenciw, anciennement de l'Agence de la santé publique du Canada (ASPC), pour ses conseils d'expert sur la surveillance du cancer et pour son examen du manuscrit. Nous remercions également Dianne Zakaria, $\mathrm{Ph}$. D., de l'ASPC, pour les discussions sur la méthode d'analyse, le $\mathrm{D}^{\mathrm{r}}$ Shiliang Liu pour les discussions sur les facteurs de risque, et $\mathrm{M}^{\mathrm{me}}$ Judy Snider pour l'examen du manuscrit.

\section{Conflits d'intérêts}

Les auteurs déclarent n'avoir aucun conflit d'intérêts.

\section{Contributions des auteurs et avis}

Tous les auteurs ont contribué à la conception de l'étude, à l'interprétation des données et à la rédaction ou à la révision de l'article. LX a effectué l'analyse.

Le contenu de l'article et les points de vue qui y sont exprimés n'engagent que les auteurs et ne reflètent pas nécessairement ceux du gouvernement du Canada.

\section{Références}

1. Comité consultatif de la Société canadienne du cancer. Statistiques canadiennes sur le cancer 2015. Toronto (Ont.) : Société canadienne du cancer; 2015.
2. Statistique Canada. Principales causes de décès, population totale, selon le groupe d'âge et le sexe, Canada [tableau CANSIM 102-0561]. Ottawa (Ont.) : Statistique Canada; 2017. En ligne à : http://www5.statcan.gc.ca /cansim/a26?lang $=$ fra\&retrLang $=$ fra $\&=1020561 \&$ tabMode $=$ dataTable $\&$ p 1 $=-1 \& \mathrm{p} 2=9 \& \operatorname{srchLan}=-1$

3. Société canadienne du cancer et Institut national du cancer du Canada. Statistiques canadiennes sur le cancer 2008. Toronto (Ont.) : Société canadienne du cancer et Institut national du cancer du Canada; 2008.

4. Ellison L, Janz T. Incidence du cancer et mortalité par cancer chez les enfants au Canada. Coup d'œil sur la santé. Ottawa (Ont.) : Statistique Canada; 2015 [no 82-624-X au catalogue]. En ligne à : http://www.statcan .gc.ca/pub/82-624-x/2015001/article /14213-fra.pdf

5. Linabery AM, Ross JA. Trends in childhood cancer incidence in the U.S. (1992-2004). Cancer. 2008;112(2):416432. doi: $10.1002 /$ cncr.23169.

6. Baade PD, Youlden DR, Valery PC, et al. Trends in incidence of childhood cancer in Australia, 1983-2006. Br J Cancer. 2010;102(3):620-626. doi: 10.1038/sj.bjc.6605503.

7. Kaatsch P. Epidemiology of childhood cancer. Cancer Treat Rev. 2010;36(4): 277-85. doi: 10.1016/j.ctrv.2010.02.003.

8. Liu YL, Lo WC, Chiang CJ, et al. Incidence of cancer in children aged 0-14 years in Taiwan, 1996-2010. Cancer Epidemiol. 2015;39(1):21-28. doi: 10.1016/j.canep.2014.11.010.

9. Statistique Canada. Registre canadien du cancer (RCC) [Internet]. Ottawa (Ont.) : Statistique Canada. En ligne à : http://www23.statcan.gc.ca/imdb /p2SV_f.1?Function = getSurvey\&SDDS $=3207$

10. Fritz A, Percy C, Jack A, et al. Classification internationale des maladies pour l'oncologie (3e éd.). Genève : Organisation mondiale de la santé; 2008. En ligne à : http://apps.who .int/iris/bitstream/10665/43859/1 /9789242545340_fre.pdf
11. Steliarova-Foucher E, Stiller C, Lacour $\mathrm{B}$, et al. International Classification of Childhood Cancer, third edition. Cancer. 2005;103(7):1457-1467. doi: 10.1002 /cncr.20910.

12. National Cancer Institute's Surveillance, Epidemiology, and End Results Program. Main Classification Table from the ICCC-3 based on ICD-O-3 [Internet]. En ligne à : https://seer .cancer.gov/iccc/iccc3.html

13. Statistique Canada. Estimations démographiques annuelles : Canada, provinces et territoires, 2015 (Tableau CANSIM 051-0001) [Internet]. Ottawa (Ont.) : Statistique Canada; 2015 [no 91-215-X au catalogue]. En ligne à : http://www.statcan.gc.ca/pub/91 -215-x/91-215-x2015000-fra.pdf

14. National Cancer Institute . Joinpoint Regression Program, Version 4.3.1.0 April 2016. Bethesda, MD : Statistical Methodology and Applications Branch, Surveillance Research Program, National Cancer Institute.

15. Kim HJ, Fay MP, Feuer EJ, et al. Permutation tests for joinpoint regression with applications to cancer rates. Stat Med. 2000;19(3):335-351. Erratum in: Stat Med 2001;20(4):655.

16. Steliarova-Foucher E, Colombet M, Ries LAG, et al. International incidence of childhood cancer, 2001-10: a population-based registry study. Lancet Oncol. 2017;18(6):719-731. doi: 10.1016 /S1470-2045(17)30186-9.

17. Siegel DA, King J, Tai E, et al. Cancer incidence rates and trends among children and adolescents in the United States, 2001-2009. Pediatrics. 2014; 134(4):e945-955. doi: 10.1542/peds .2013-3926.

18. Huchcroft S, Clarke A, Y Mao, et al. Mon combat pour la vie : le cancer chez les enfants et les adolescents au Canada. Ottawa (Ont.) : Approvisionnements et Services Canada; 1996.

19. Neglia JP, Friedman DL, Yasui Y, et al. Second malignant neoplasms in fiveyear survivors of childhood cancer: childhood cancer survivor study. J Natl Cancer Inst. 2001;93(8):618-629. doi: $10.1093 /$ jnci/93.8.618. 
20. Johnson KJ, Carozza SE, Chow EJ, et al. Parental age and risk of childhood cancer: a pooled analysis. Epidemiology. 2009;20(4):475-483. doi: 10.1097/EDE .0b013e3181a5a332.

21. Statistique Canada. Fécondité : moins d'enfants, mères plus âgées [Internt]. Ottawa (Ont.) : Statistique Canada; [modification le 29 sept. 2016]. En ligne à : http://www.statcan.gc.ca/pub/11 -630-x/11-630-x2014002-fra.htm

22. Gittleman HR, Ostrom QT, Rouse CD, et al. Trends in central nervous system tumor incidence relative to other common cancers in adults, adolescents, and children in the United States, 2000 to 2010. Cancer. 2015;121(1):102-112. doi: $10.1002 /$ cncr.29015.

23. Eden T. Aetiology of childhood leukaemia. Cancer Treat Rev. 2010;36(4):286297. doi: 10.1016/j.ctrv.2010.02.004.

24. Lavige É, Bélair MA, Do MT, et al. Maternal exposure to ambient air pollution and risk of early childhood cancers: A population-based study in Ontario, Canada. Environ Int. 2017; 100:139-147. doi: 10.1016/j.envint.2017 .01 .004 .

25. Carlos-Wallace FM, Zhang L, Smith MT, et al. Parental, In Utero, and Early-Life Exposure to Benzene and the Risk of Childhood Leukemia: A Meta-Analysis. Am J Epidemiol. 2016; 183(1):1-14. doi: 10.1093/aje/kwv120.

26. Chen M, Chang $\mathrm{CH}$, Tao L, et al. Residential exposure to pesticide during childhood and childhood cancers: a meta-analysis. Pediatrics. 2015;136(4): 719-729. doi: 10.1542/peds.2015-0006.

27. Ross JA, Swensen AR. Prenatal epidemiology of pediatric tumors. Curr Oncol Rep. 2000;2(3):234-241.

28. Linet MS, Brown LM, Mbulaiteye SM, et al. International long-term trends and recent patterns in the incidence of leukemias and lymphomas among children and adolescents ages 0-19 years. Int J Cancer. 2016;138(8):18621874. doi: 10.1002/ijc.29924.

29. Torabi M, Singh H, Galloway K, et al. Geographical variation in the incidence of childhood leukaemia in Manitoba. J Paediatr Child Health. 2015;51(11): 1121-1126. doi: 10.1111/jpc.12930.
30. Statistique Canada. 150 ans d'immigration au Canada. Ottawa (Ont.) : Statistique Canada; 2016. En ligne à : https://www.statcan.gc.ca/pub/11-630 -x/11-630-x2016006-fra.htm

31. Jonathan Chagnon. Migrations internationales, 2010 et 2011. Ottawa (Ont.) : Statistique Canada; 2013 [no 91-209-X au catalogue]. En ligne à : http://www.statcan.gc.ca/pub/91209-x/2013001/article/11787-fra.pdf

32. Statistique Canada. Tendances du recensement : Choix relatif à la géographie : Canada, provinces et territoires [Internet]. Ottawa (Ont.) : Statistique Canada; 2007. En ligne à : http://www12.statcan.gc.ca/census -recensement/2006/dp-pd/92-596/P1-2 .$c f m ?$ Lang $=$ fra $\& \mathrm{~T}=\mathrm{PR} \& \mathrm{PRCODE}=35 \&$ $\mathrm{GEOCODE}=35 \& \mathrm{GEOLVL}=\mathrm{PR} \& \mathrm{TID}=800$

33. McKean-Cowdin R, Razavi P, Barrington-Trimis $\mathrm{J}$, et al. Trends in childhood brain tumor incidence, 1973-2009. J Neurooncol. 2013;115(2): 153-60. doi: 10.1007/s11060-013-1212-5.

34. Papathoma P, Thomopoulos TP, Karalexi MA, et al. Childhood central nervous system tumours: incidence and time trends in 13 Southern and Eastern European cancer registries. Eur J Cancer. 2015;51(11):1444-1455. doi: 10.1016/j.ejca.2015.04.014.

35. Centre International de Recherche sur le Cancer. Agents classés par les Monographies du CIRC, volumes 1-109 - Monographies du CIRC sur l'évaluation des risques de cancérogénicité pour l'homme [Internet]. Lyon (FR) : Centre International de Recherche sur le Cancer; 2013. En ligne à : http:// monographs.iarc.fr/FR/Classification /index.php

36. Boyle P, Levin B (dir.). World Cancer report 2008 [Internet]. Lyon (FR) : International Agency for Research on Cancer; 2008. En ligne à : http://www .iarc.fr/en/publications/pdfs-online /wcr/2008/wcr_2008.pdf

37. Kaatsch P, Steliarova-Foucher E, Crocetti E, et al. Time trends of cancer incidence in European children (19781997): report from the Automated Childhood Cancer Information System project. Eur J Cancer. 2006;42(13): 1961-1971. doi: 10.1016/j.ejca.2006.05 .014 .
38. Spector LG, Birch J. The epidemiology of hepatoblastoma. Pediatr Blood Cancer. 2012;59(5):776-779. doi: 10.1002 /pbc. 24215 .

39. McLaughlin CC, Baptiste MS, Schymura MJ, et al. Maternal and infant birth characteristics and hepatoblastoma. Am J Epidemiol. 2006;163(9):818-828. doi: 10.1093/aje/kwj104.

40. Ansell P, Mitchell CD, Roman E, et al. Relationships between perinatal and maternal characteristics and hepatoblastoma: a report from the UKCCS. Eur J Cancer. 2005;41(5):741-748. doi: 10.1016/j.ejca.2004.10.024.

41. Spector LG, Feusner JH, Ross JA. Hepatoblastoma and low birth weight. Pediatr Blood Cancer. 2004;43(6):706. doi: 10.1002/pbc.20122.

42. Agence de la santé publique du Canada. Indicateurs de la santé périnatale au Canada 2013 : un rapport du Système canadien de surveillance périnatale. Ottawa (Ont.) : Agence de la santé publique du Canada; 2013. En ligne à : http://publications.gc.ca /collections/collection_2014/aspc -phac/HP7-1-2013-fra.pdf

43. Larrañaga N, Sanchez MJ, Ardanaz E, et al. Incidence Patterns and Trends of non-Central Nervous System Solid Tumours in Children and Adolescents. A Collaborative Study of the Spanish Population Based Cancer Registries. J Cancer. 2016;7(3):335-343. doi: 10.7150 /jca.12943.

44. Park JR, Eggert A, Caron H. Neuroblastoma: biology, prognosis, and treatment. Hematol Oncol Clin North Am. 2010;24(1):65-86. doi: 10.1016/j .hoc.2009.11.011

45. Woods WG, Gao Rn, Shuster JJ, et al. Screening of infants and mortality due to neuroblastoma. $\mathrm{N}$ Engl $\mathrm{J}$ Med. 2002;346(14):1041-1046. doi: 10.1056/ NEJMoa012387.

46. Holmes L Jr, Hossain J, Opara F. Pediatric thyroid carcinoma incidence and temporal trends in the USA (19732007): race or shifting diagnostic paradigm? ISRN Oncol. 2012;2012:906197. doi: $10.5402 / 2012 / 906197$. 
47. Xie L, Semenciw R, Mery L. Incidence du cancer au Canada : tendances et projections (1983-2032). Promotion de la santé et prévention des maladies chroniques au Canada. 2015;35 (suppl. 1):2-196. doi: 10.24095/hpcdp.35.S1 $.02 \mathrm{f}$.

48. Siegel R, Ma J, Zou Z, et al. Cancer statistics, 2014. CA Cancer J Clin. 2014;64(1):9-29. doi: 10.3322/caac .21208 .

49. Kent WD, Hall SF, Isotalo PA, et al. Increased incidence of differentiated thyroid carcinoma and detection of subclinical disease. CMAJ. 2007;177(11): 1357-61. doi: 10.1503/cmaj.061730.

50. Linet MS, Kim KP, Rajaraman P. Children's exposure to diagnostic medical radiation and cancer risk: epidemiologic and dosimetric considerations. Pediatr Radiol. 2009;39 (Suppl 1):S4-26. doi: 10.1007/s00247-008 -1026-3.

51. Hammer GP, Seidenbusch MC, Regulla $\mathrm{DF}$, et al. Childhood cancer risk from conventional radiographic examinations for selected referral criteria: results from a large cohort study. AJR Am J Roentgenol. 2011;197(1):217223. doi: 10.2214/AJR.10.4979.

52. Schonfeld SJ, Lee C, Berrington de González A. Medical exposure to radiation and thyroid cancer. Clin Oncol (R Coll Radiol). 2011;23(4):244-50. doi: 10.1016/j.clon.2011.01.159.

53. Peterson E, De P, Nuttall R. BMI, diet and female reproductive factors as risks for thyroid cancer: a systematic review. PLoS One. 2012;7(1):e29177. doi: 10.1371/journal.pone.0029177.

54. Meinhold CL, Ron E, Schonfeld SJ, et al. Nonradiation risk factors for thyroid cancer in the US Radiologic Technologists Study. Am J Epidemiol. 2010;171(2):242-52. doi: 10.1093/aje /kwp354.

55. Statistique Canada. Tendances de la santé 2013 [Internet]. Ottawa (Ont.) : Statistique Canada; 2013 [no 82-213XWF au catalogue]. En ligne à : http:// www12.statcan.gc.ca/health-sante/82 $-213 /$ index.cfm? Lang $=$ fra
56. Agence de la santé publique du Canada. Obésité au Canada [Internet]. Ottawa (Ont.) : Agence de la santé publique du Canada; 2011. En ligne à : https://www.canada.ca/fr/sante -publique/services/promotion-sante /modes-vie-sains/obesite-canada /adultes.html\#figure-1

57. Tjepkema, M. Obésité chez les adultes au Canada : poids et grandeur mesurés. Dans : Nutrition : résultats de l'Enquête sur la santé dans les collectivités canadiennes, no 1. Ottawa (Ont.) : Statistique Canada; 2006 [no 82-620-MWF au catalogue].

58. Irvine B, Luo W, León JA. Anomalies congénitales au Canada 2013 : rapport de surveillance sur la santé périnatale du Système canadien de surveillance périnatale de l'Agence de la santé publique du Canada. Promotion de la santé et prévention des maladies chroniques au Canada. 2015;35(1):25-26. 